\title{
Tail resonances of FPU q-breathers and their impact on the pathway to equipartition
}

\author{
Tiziano Penati* \\ Dipartimento di Matematica "F.Enriques" \\ Via Saldini 50, 20133 Milano, Italy \\ Sergej Flach \\ Max-Planck-Institut für Physik komplexer Systeme \\ Nöthnitzer Str. 38, 01187 Dresden, Germany
}

3rd July 2018

\begin{abstract}
Upon initial excitation of a few normal modes the energy distribution among all modes of a nonlinear atomic chain (the Fermi-PastaUlam model) exhibits exponential localization on large time scales. At the same time resonant anomalies (peaks) are observed in its weakly excited tail for long times preceding equipartition. We observe a similar resonant tail structure also for exact time-periodic Lyapunov orbits, coined q-breathers due to their exponential localization in modal space. We give a simple explanation for this structure in terms of superharmonic resonances. The resonance analysis agrees very well with numerical results and has predictive power. We extend a previously developed perturbation method, based essentially on a PoincaréLindstedt scheme, in order to account for these resonances, and in order to treat more general model cases, including truncated Toda potentials. Our results give qualitative and semiquantitative account for the superharmonic resonances of q-breathers and natural packets.
\end{abstract}

*corresponding author: tiz77@yahoo.it 
The Fermi-Pasta-Ulam model (FPU) is a one dimensional nonlinear lattice whose dynamics, for small energy densities $\mathcal{E}$ and initially localized (in Fourier space) excitations, is characterized by a long time freezing of the harmonic energy among a subset of normal modes (natural packets). This metastable regime persists until, after times growing exponentially in $\frac{1}{\mathcal{E}}$, equipartition is reached. The intermediate state is characterized by an exponential localization of the harmonic energies around the initially excited modes and the appearance of resonant deviations in the tail of the energy distribution. During the transition from metastability to stochasticity, redistribution of mode energies happens through the tail resonances.

The system admits also exact periodic orbits - $q$-breathers (QB) - which are obtained by continuation of the normal modes of the linear system. QBs also show both exponential localization of mode energy distribution and resonant tail structures. The QB localization holds below an energy density threshold $\mathcal{E}^{*}$ which depends on the nonlinear parameter. The tail resonances are due to small denominators which appear naturally in a Poincare-Lindstedt perturbation scheme.

We observe that the dynamics of the nonlinear FPU model generated by initially exciting one normal mode (FPU trajectory) exhibits the main elements of the nearby $q$-breather. Thus the presented analytical approach, besides describing the $q$-breather, also explains some relevant features of the corresponding FPU trajectory. Remarkably, this correspondence may hold on very long times, which requires further rigorous explanations.

\section{Introduction}

\subsection{Brief history}

The computational experiments which Fermi, Pasta and Ulam performed around 1950 in the Los Alamos laboratory are nowadays considered as an important step in the progress of understanding nonlinear dynamics. The main aim of their numerical investigation was to observe, as they expected, a "relatively fast" and "equally distributed" sharing of the averaged harmonic energy of an anharmonic atomic chain among all the normal modes, even when starting with initial conditions far from some equipartition regime. When chosing initial data localized on just one low frequency mode they however did not observe any actual equipartition, but a localization of the energy among some initial modes in the nearby frequency sector (also coined in the 
literature and hereafter natural packets), including some additional almost complete recurrence to the initially excited mode.

Among the several explanations that followed, we recall the one given in 1965 by Izrailev and Chirikov 23, 24. They suggested the idea that the KAM theorem was the right approach in order to explain the regular dynamical behaviour observed: if the energy given to the system is below a threshold $E^{*}(N)$, then the dynamics is influenced by the persistence of most of the nonresonant invariant tori. Since $\lim _{N \rightarrow \infty} \mathcal{E}^{*}=0$, where $\mathcal{E}^{*}=\frac{E^{*}(N)}{N}$ is the energy density, the FPU phenomenon should not be relevant in the thermodynamic limit at any finite temperature or energy density. In 1970, instead, Bocchieri, Scotti, Bearzi and Loinger [5] conjectured the existence of a specific energy threshold $\mathcal{E}^{*}$ (which remains positive in the thermodynamic limit) below which equipartition is not reachable. Recent numerical results [3, 4, 27 indicate that below that threshold equipartition is still reached for infinite times, yet the time scale of the route to equipartition may diverge exponentially with lowering the energy density.

Several computational experiments have been performed (e.g. [11, 10, 35, 3, 4, 21), suggesting possible answers about the time needed to approach equipartition, its scaling with the energy density and dimension of the system and the features of the dynamics during this transition. Only few analytical studies touch the case of large systems: here we mention some relevant results related to the application of the KAM Theorem [31, 33], to some special (periodic) solutions [29, 32] and to a normal form construction via a PDE [1].

Recently Flach, Ivanchenko and Kanakov [16] have focussed on the main FPU observation that the initially excited normal mode shares its energy for long times only with a few other modes from a frequency neighbourhood in modal space. They have identified this long lasting regime as a dynamical localization effect and applied the methods developed for discrete breathers in FPU chains [15] to the dynamics of normal modes. The result is that q-breathers (QB) - time-periodic and modal-space-localized orbits - persist in the FPU model. The dynamics generated by one initially excited mode evolves close to the related q-breathers for very long times. Thus many features of the short- and medium-time evolution of natural packets are encoded in the profile of a $\mathrm{QB}$ and the phase space flow of small fluctuations around a given QB.

\subsection{The FPU model}

Fermi, Pasta and Ulam considered a non-linear one-dimensional lattice, with fixed boundary conditions

$$
\left\{\begin{array}{l}
H(\underline{x}, \underline{y})=\frac{1}{2} \sum_{j=1}^{N} y_{j}^{2}+\sum_{j=0}^{N} V\left(x_{j+1}-x_{j}\right) \\
x_{0}=x_{N+1}=0
\end{array}\right.
$$

where $V(r)=\frac{1}{2} r^{2}+\frac{\alpha}{3} r^{3}+\frac{\beta}{4} r^{4}$ is the coupling potential, $\left(x_{j}, y_{j}\right)$ are respectively the displacement from the equilibrium of the j-particle and its kinetic momentum, while the parameters $\alpha, \beta$ may be considered 
as real and positive. The case of linear equations of motion, given by the quadratic Hamiltonian

$$
H_{0}(\underline{x}, \underline{y})=\frac{1}{2} \sum_{j=0}^{N}\left[y_{j}^{2}+\left(x_{j+1}-x_{j}\right)^{2}\right]
$$

is integrable and corresponds to a system of $N$ uncoupled normal mode oscillators. We can show this via the change of coordinates

$$
\begin{aligned}
Q_{l} & =\sqrt{\frac{2}{N+1}} \sum_{j=1}^{N} x_{j} \sin \left(\frac{j l \pi}{N+1}\right) \\
P_{l} & =\sqrt{\frac{2}{N+1}} \sum_{j=1}^{N} y_{j} \sin \left(\frac{j l \pi}{N+1}\right)
\end{aligned}
$$

which diagonalizes the Hamiltonian $H_{0}$

$$
H_{0}(\underline{Q}, \underline{p})=\frac{1}{2} \sum_{l=1}^{N}\left(P_{j}^{2}+\omega_{j}^{2} Q_{j}^{2}\right)
$$

where $\left(Q_{j}, p_{j}\right)$ are the normal mode coordinates and momenta, and $\omega_{j}$ are the normal mode frequencies given by

$$
\omega_{j}=2 \sin \left(\frac{j \pi}{2 N+2}\right)
$$

The effect of the non-linear part in the model (11) is to introduce an interaction between the different normal mode oscillators. In this way they exchange energy and the harmonic actions are in general no longer preserved. Even if the considered change to normal modes appears hard to develop for the cubic and quartic part of the Hamiltonian, there is a mathematical way to exhibit which are the selection rules that couple the different oscillators (see also [10]). Let us consider the so called $\alpha$-model, where $\beta=0$. The cubic part of the potential can be rewritten as

$$
\frac{\alpha}{3} \sum_{j=0}^{N}\left(x_{j+1}-x_{j}\right)^{3}=\frac{\alpha}{3 \sqrt{2 N+2}} \sum_{1 \leq i, j, h \leq N} \omega_{i} \omega_{j} \omega_{h} Q_{i} Q_{j} Q_{h} B_{i j h}
$$

where we set $B_{i j h}=\sum_{\sigma_{1,2}= \pm 1} \Delta_{i+\sigma_{1} j+\sigma_{2} h}$, with

$$
\Delta_{i \pm j \pm h}=\left\{\begin{array}{cc}
1 & , i \pm j \pm h=0 \\
-1 & , i \pm j \pm h=2(N+1) \\
0 & , \text { otherwise }
\end{array}\right.
$$



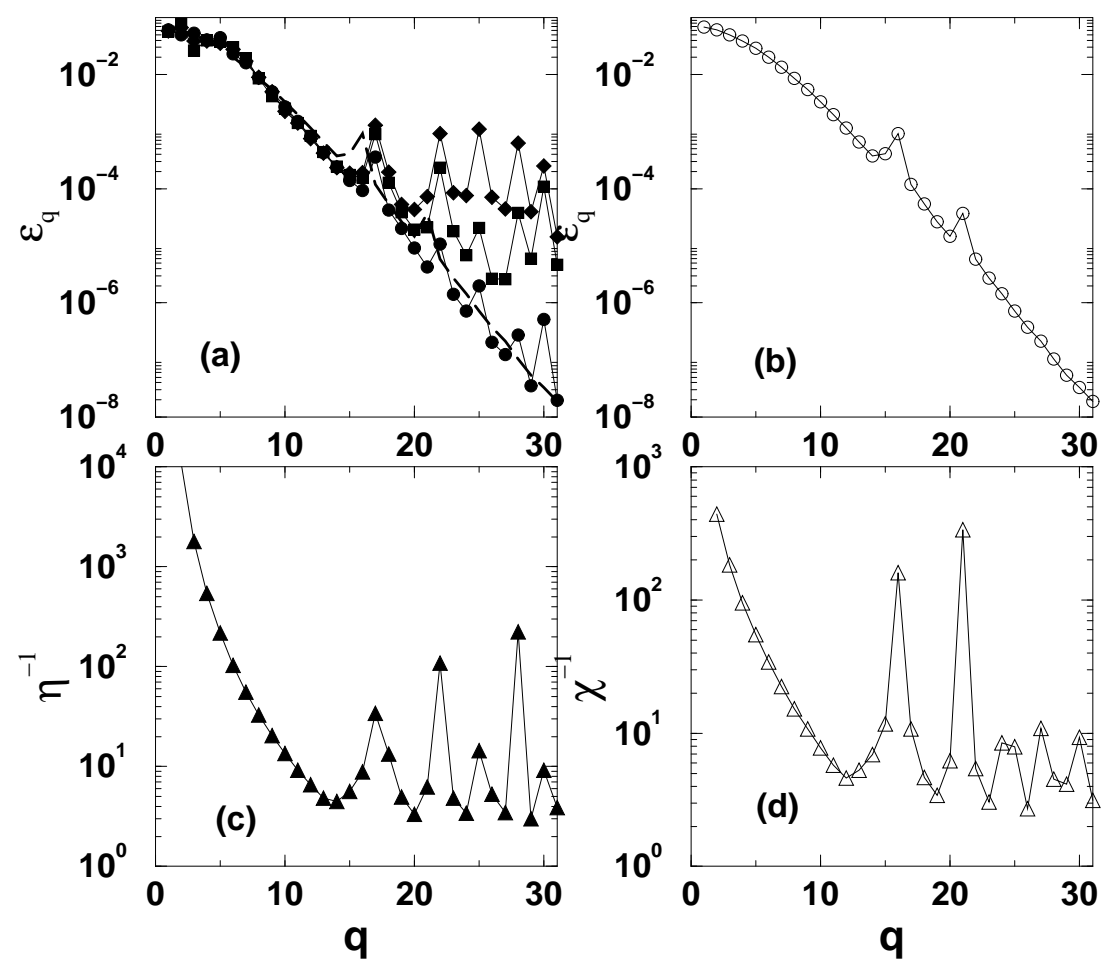

Figure 1: (a): Natural packet evolution initially exciting mode $q_{0}=1$. The energies of normal modes are plotted versus $q$. Circles $-10^{4}$, squares $10^{5}$, rhombs - $10^{6}$. Dashed line - $q$-breather from (b) for comparison. (b): The energies of normal modes versus $q$ for a $q$-breather with $q_{0}=1$. (c): sequence $\eta_{j}^{-1}$. (d): sequence $\chi_{j}^{-1}$. Parameters in all cases: $N=31, \alpha=$ $0.33, \mathcal{E}=0.01$.

The equations of motion for a normal mode oscillator then read

$$
\ddot{Q}_{j}+\omega_{j}^{2} Q_{j}=-\frac{\alpha}{\sqrt{2(N+1)}} \sum_{1 \leq i, h \leq N} \omega_{j} \omega_{i} \omega_{h} Q_{i} Q_{h} B_{i j h}
$$

\subsection{Natural packets}

One of the findings which can be related to Bocchieri's conjecture is the so called 'metastability' of natural packets, which has been numerically investigated by A.Giorgilli and others [3, 4, 21, 27. What has been observed in [3] is that, for energy density small enough, an initial excitation of the first mode is spread among nearby modes up to a natural frequency which depends only on the energy density, according to the power law $\omega \sim \mathcal{E}^{1 / 4}$. This localization of the harmonic energy on a subset of modes, which can coexist with chaotic dynamics inside the subset, persists for times which can grow exponentially with the inverse of the energy density (see [4]), and only after this long 

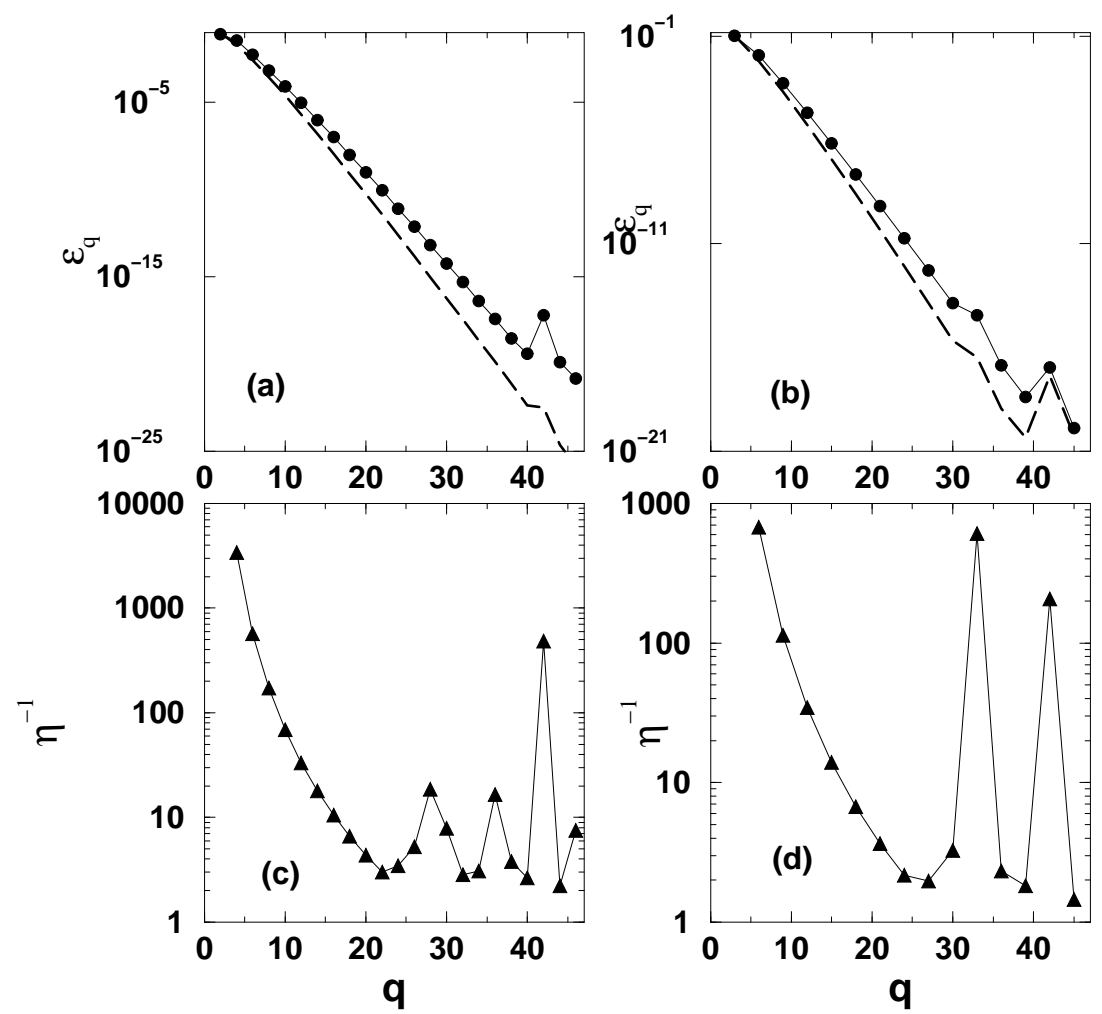

Figure 2: (a): Natural packet evolution initially exciting mode $q_{0}=2$ (circles). The energies of normal modes are plotted versus $q$ at $t=10^{5}$. The dashed line shows the energy distribution for a corresponding $q$-breather with $q_{0}=2$. (b): Same as (a), but for $q_{0}=3$. (c): sequence $\eta_{j}^{-1}(2,47)$. (d): sequence $\eta_{j}^{-1}(3,47)$. Parameters in all cases: $N=47, \alpha=0.1, \mathcal{E}=0.0025$. 
transient the system reaches a proper equipartition regime. The timedependence of the size of these natural packets of normal modes show two time scales $\tau_{1,2}$ which separate strongly when the energy density is below a certain threshold. The first time scale $\tau_{1}$ characterizes the time needed for the formation of a natural packet of nearly fixed (and quite localized) shape in normal mode space. The second time scale $\tau_{2}$, which becomes much larger compared to the first one when the energy density is lowered, characterizes the time needed to finally delocalize the packet over all modes and to reach equipartition. Both time scales $\tau_{1} \approx \tau_{2}$ at some energy density threshold. Thus at and above the energy density threshold no metastable or intermediate normal mode localization occurs, and the natural packet spreads more or less continuously in time over all modes. Further numerical simulations [4] used the spectral entropy indicator (which gives the effective fraction of modes involved in the dynamics) and showed that the second time scale nicely fits with an exponential law.

An example of the evolution of such a packet for energy densities below the threshold (when $\tau_{1}<10^{4}, \tau_{2}>10^{9}$ ) was observed in 21] (Fig.3 therein). Following that work, we performed a similar simulation, see Fig 1a). The normal mode $q_{0}=1$ has been initially excited, and the harmonic energy distribution is shown for three different times $t=10^{4}, 10^{5}, 10^{6}$. Since harmonic mode energies may fluctuate, we plot the time-averaged quantities, with a time-averaging window of $10^{4}$. The logarithmic scale reveals that for $t=10^{4}$ the overall strong exponential distribution, which is locked over many decades in time, shows pronounced resonant peaks in the far tails (note however that even in the peak maxima the mode energies are orders of magnitude smaller than the energies of the main modes excited). At $t=10^{5}$ a gradual change is observed - the resonant peaks in the tail slowly grow and drag the tail upwards. At $t=10^{6}$, approaching $\tau_{2}$, this process continues and the slow but steady spreading of the packet over all modes can be already anticipated. At even larger times, the harmonic energy becomes equally distributed over all the normal modes (not displayed here).

In Fig. 2 (a,b) instead, the initial excitation is localized on the second and third mode, respectively. The profiles are shown for time $\tau_{1}<t=10^{5}<\tau_{2}$. They are again characterized by the existence of peaks in the tails of the distributions. The persistence of these anomalies when changing the initial mode number excited, suggests that there is a robust and generic pathway of equipartition which goes via excitation of these resonant tail peaks.

Let us compare these results to the profiles of corresponding $q$ breathers [16. We compute periodic orbits, which correspond to only one normal mode with $q_{0}$ being excited in the limit of vanishing nonlinearity. In Fig (b) the profile of a $q$-breather with $q_{0}=1$ is shown. The harmonic normal mode energies are averaged over one period of the $q$-breather. We observe exponential localization, and in the tail two distinct resonant peaks. For comparison we plot the same result as a dashed line in Fig पa). The exponential profiles of the $q$-breather and 
the corresponding natural packet are strikingly similar in the whole $q$ space. That supports previous results that a natural packet for $t \ll \tau_{2}$ evolves very close to a corresponding $q$-breather orbit. The tail resonance locations though differ for both trajectories. That signals that the nature of the tail resonances can be understood already by studying a $q$-breather, but their location is perhaps very sensitive to small perturbations. Nevertheless some common features are also observed - the tail resonances start to appear in the second part of the $q$-space. The dashed lines in Fig 2(a,b) are $q$-breather profiles for corresponding smaller energies. We note that here not only the exponential decay fits well with the natural packets, but now also the location and even the strength of the tail resonances agrees well with the one of the natural packets. We conclude that the correspondence between natural packets and $q$-breathers improves with lowering the energy also with respect to the sensitive tail resonance structures.

Further similarity between natural packets and $q$-breathers follows from 22 where it was shown, that the localization length of a QB depends on the expansion parameters of the potentials, the energy density $\mathcal{E}$ and the normalized wave number $\mathbf{k}_{\mathbf{0}}=q_{0} / N$, but not anymore on the system size $N$. In fact QBs can be scaled to and obtained in infinitely large lattices [25]. Using estimates from perturbation theory we can conclude that delocalization of QBs will happen when the energy density exceeds some critical value (with the other relevant parameters being fixed). It can be expected then that an initially localized excitation will quickly spread over all modes as well. This is in contrast to the case when the energy density is below the QB threshold. Then the QB is exponentially localized, and an initially localized excitation is known to spread a bit and take a form similar to a QB for long times, with subsequent departure for much longer times. That gives further evidence that the exact and simple periodic orbits - q-breathers - describe the metastable features of a natural packet spreading in modal space. It is then also natural to study whether the q-breather shows similar resonant tail peak features as the natural packet which evolves close to the QB. For example, in Fig. 团 (b) the exact QB corresponding to $q_{0}=1$ shows an exponential decay which persists also in the tail, while the tail's harmonic distribution of the close orbit (Fig. 廿(a)) is more irregular. Moreover, the QB also exhibits, even if in different sites, a couple of resonant peaks.

In the following we will first (Section 2.) formulate a simple superharmonic resonance analysis and show that it accounts quantitatively for the observed tail peak positions. Then (Section 3.) we will perform a modified perturbation approach to the q-breather profile and provide a semiquantitative answer for the tail profiles. 


\section{Tail resonances}

\section{$2.1 q$-breathers}

As we noticed concluding the previous section, a fruitful way to investigate the dynamics for very localized initial excitations is to study the existence and the related properties of nearby invariant manifolds. we thus focus first on the Lyapunov orbits, called $q$-breathers due to their localization in the normal mode space. These orbits are the natural continuation, for small values of a coupling parameter, of the uncoupled oscillatory solutions which appear in the linear problem [16. The existence of these families for every linear frequency $\omega_{j}$ of (11) goes through classical continuation theorems, such as the Lyapunov Theorem or the Poincaré Theorem, and essentially relies on the property of rational independence of any couple of frequencies composing the spectrum. Indeed old (9]) and more recent (13) results on sums of roots of Unity have proved that any linear combination of two different frequencies with rational coefficients is always different from zero

$$
k_{j} \omega_{j}+k_{l} \omega_{l} \neq 0, \quad \forall k_{j}, k_{l} \in \mathbb{Q}, \quad \omega_{j} \neq \omega_{l} .
$$

In order to apply the above mentioned Theorems, it is enough to use a weaker result which ensures that (4) holds when $k_{l}=1$ and $\omega_{j}$ is the frequency of the linear periodic orbit we want to continue. The new periodic orbits are expected to be continuous deformations of the original ones upon changing the nonlinearity parameter. Thus, if we initially excite only one $q_{0}$-mode, we can imagine the dynamics being strongly influenced by the corresponding $q_{0}$-breather: in fact, the initial condition is close to this special orbit in phase space.

The idea to interpret the original results obtained by Fermi, Pasta and Ulam in terms of deformation of periodic Lyapunov orbits has been recently used in [16. In this paper the authors perform both a numerical and analytical investigation of the $\alpha$ and $\beta$ models. Their study gives an exponential localization of the energy among the modes involved in the periodic orbit; these modes are selected according to the definition of the coupling coefficients $B_{i j h}$ (see eq (2)). Furthermore, the linear stability analysis for the $\beta$ model remarkably reveals that this exponential localization, which gets weaker when increasing the coupling parameter $\beta$, is still persistent when the orbit becomes unstable. From the analytical point of view, the authors provide an application of the century old proof of Lyapunov to the existence of q-breathers, and a perturbative Poincaré-Lindstedt expansion which explains the localization for the modes $k q_{0}$ belonging to the low frequency part of the spectrum, id est when both $q_{0} \ll N$ and $k q_{0} \ll N$. This perturbation scheme also shows how, at every step $k$, only one new mode, precisely the mode $k q_{0}$, is involved; then, by using a cubic expansion of the spectrum to cope with the small divisors, the dynamics of this mode is approximated as an oscillation whose frequency is $k \omega_{q_{0}}$.

In order to better understand the idea that moved our research, we 
recall here just the main steps of their deduction, and will extend this approach in the next section. The starting point consists in expanding the periodic solution with respect to a perturbation parameter, here chosen as $\sigma=\frac{\alpha}{\sqrt{2 N+2}}$

$$
Q_{q}(t)=\sum_{j \geq 0} \sigma^{j} Q_{q}^{(j)}(t)
$$

in (3). Sorting the terms with respect to powers of $\sigma$, we get an infinite sequence of differential equations, one for each component $Q_{q}^{(j)}, j \geq 0$; the zero order solution is simply the cosinusoidal oscillation of the $q_{0}$ mode (or the linear approximation)

$$
Q_{q}^{(0)}=\delta_{q, q_{0}} A_{q_{0}} \cos \left(\omega_{q_{0}} t\right)
$$

For any $n \geq 2$, the scheme then follows these rules:

- at every step $n-1$, only one new mode $Q_{n q_{0}}^{(n-1)}(t)$ is involved in the motion. This is related to the coupling coefficients $B_{n q_{0}, l, m}$.

- up to the step $n-1$, every solution $Q_{q}^{(h)}, 0 \leq h \leq n-2, q_{0} \leq$ $q \leq(n-1) q_{0}$ is a finite sum of harmonics having frequencies $m \omega_{q_{0}}, m \leq h+1$. Among these solutions, the one $Q_{j q_{0}}^{(j-1)}(t), j=$ $2, \ldots, n-1$ are approximated with the largest amplitude oscillation. Therefore the new forced oscillator becomes

$$
\frac{d^{2}}{d t^{2}} Q_{n q_{0}}^{(n-1)}+\omega_{n q_{0}}^{2} Q_{n q_{0}}^{(n-1)}=\sum_{m=0}^{n} C_{m} \cos \left(m \omega_{q_{0}} t\right),
$$

with a suitably defined sequence $C_{m}$; the initial conditions are chosen such that the solution reads

$$
Q_{n q_{0}}^{(n-1)}=\sum_{m=0}^{n} \frac{C_{m}}{\omega_{n q_{0}}^{2}-m^{2} \omega_{q_{0}}^{2}} \cos \left(m \omega_{q_{0}} t\right) .
$$

- exploiting the cubic expansion at low frequencies $\omega_{k} \approx k \delta-$ $\frac{1}{24} k^{3} \delta^{3}, \delta=\frac{\pi}{N+1}$, it is possible both to realize that the smallest divisor is $\omega_{n q_{0}}^{2}-n^{2} \omega_{q_{0}}^{2}$ and to give an approximated value of this quantity as

$$
\omega_{n q_{0}}^{2}-n^{2} \omega_{q_{0}}^{2} \approx-\frac{1}{12} n^{2}\left(n^{2}-1\right) \omega_{q_{0}}^{4} .
$$

Hence, the solution can still be reduced to a single oscillator

$$
Q_{n q_{0}}^{(n-1)}=\frac{12 C_{n}}{n^{2}\left(n^{2}-1\right) \omega_{q_{0}}^{4}} \cos \left(n \omega_{q_{0}} t\right)\left(1+\mathcal{O}\left(\frac{n q_{0}}{N}\right)^{2}\right)
$$

if the error $\mathcal{O}\left(\frac{n q_{0}}{N}\right)^{2}$ is small. 

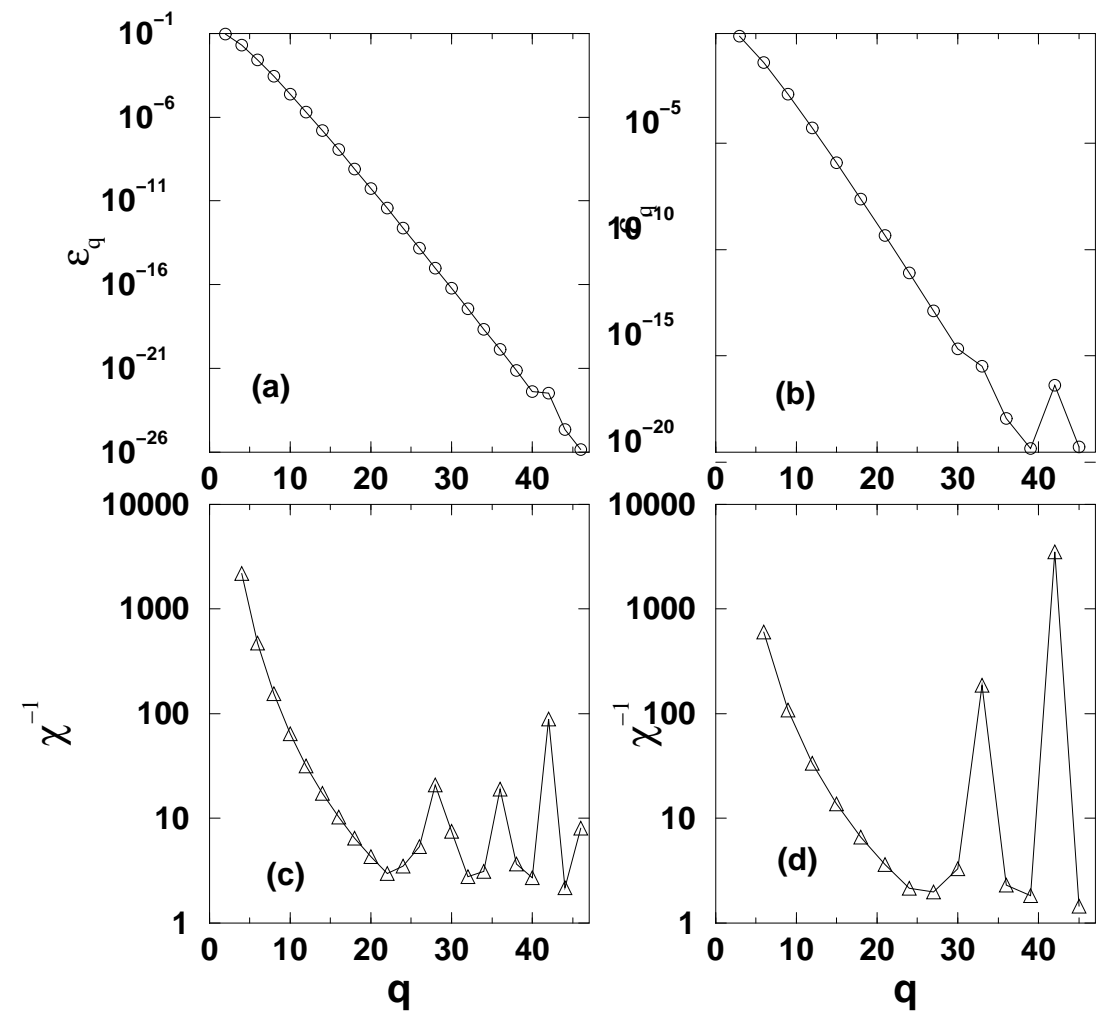

Figure 3: Parameters: $N=47, \alpha=0.1, \mathcal{E}=0.0025$. (a): Energy of normal modes versus mode number of a QB for $q_{0}=2$. (b): Similar to (a), but for a QB with $q_{0}=3$. (c): sequence $\chi_{j}^{-1}(2,47)$. (d): sequence $\chi_{j}^{-1}(3,47)$.

\section{$2.2 \quad$ Higher order nonlinear resonances}

The perturbation scheme used in 16 is based on the cubic approximation of the dependence of $\omega_{j}$ on $j$. This approximation holds in the low frequency domain and allows to perform a detailed analysis of the small divisors. In this way one limits the study to the first third of the spectrum from below, were this approximation holds. However, the numerical computation of these orbits clearly shows an exponential decay which continues into the upper two thirds of the normal mode frequency spectrum. However, some of these orbits also show resonant deviations in the tails. We conjecture that these resonances are due to almost resonances which are not captured by the abovementioned cubic approximation of the dependence of normal mode frequencies on mode numbers. Thus we investigate the sequence of small divisors

$$
\bar{\eta}_{n, m}=\left|\omega_{n q_{0}}^{2}-m^{2} \omega_{q_{0}}^{2}\right| \quad m=n, n-2, n-4, \ldots
$$

for every frequency $\omega_{n q_{0}}$ belonging to a mode involved in the $q$-breather considered. The special choice of only odd or even multiples is related 
to the perturbation theory and will become clear later. Then, for each $n$, we can also consider the sequence of the smallest divisors

$$
\eta_{n}=\min _{m=n, n-2, \ldots}\left\{\bar{\eta}_{n, m}\right\} .
$$

For each $q_{0}$, the above definitions refers to $\omega_{q_{0}}$, which is the first order approximation of the true frequency $\Omega_{q_{0}}$ of the $q_{0}$-breather considered. Equations (8) and (9) are a consequence of applying the PoincaréLindstedt method, since these are the small denominators appearing in the Fourier expansion of the leading solution (6). However the approximating $\Omega_{q_{0}}$ by $\omega_{q_{0}}$ is less accurate the larger we take the product of perturbation parameters $\alpha \mathcal{E}$. Thus intuition suggests to consider also sequences obtained by replacing $\omega_{q_{0}}$ with the true QB frequency $\Omega_{q_{0}}(\alpha, \mathcal{E})$

$$
\bar{\chi}_{n, m}=\left|\omega_{n q_{0}}^{2}-m^{2} \Omega_{q_{0}}^{2}\right|, \quad \chi_{n}=\min _{m=n, n-2, \ldots}\left\{\bar{\chi}_{n, m}\right\},
$$

with the same choice of $m$. These are the small denominators appearing in a pure Fourier expansion of the solutions $Q_{n q_{0}}$ by substituting $Q_{n q_{0}}=\sum_{k \in \mathbb{Z}} \hat{Q}_{n q_{0}, k} e^{i k \Omega_{q_{0}} t}$ in (3).

In Fig प(c,d) we plot the sequences $\eta_{n}^{-1}$ and $\chi_{n}^{-1}$ for $q_{0}=1, N=31$ and $\mathcal{E}=0.01$, and in Fig. 2(c,d) and Fig $3(\mathrm{c}, \mathrm{d})$ respectively the sequences $\eta_{n}^{-1}$ and $\chi_{n}^{-1}$ for $q_{0}=2,3, N+1=48, \alpha=0.1$ and $\mathcal{E}=0.0025$. Note that the corresponding true QB frequency $\Omega_{q_{0}}$ has been used to compute $\chi$. In all cases the first part of the sequence is influenced by the cubic approximation. The curves follow the law $\frac{1}{3 \log (k \delta)}$ and the largest term corresponds to the smallest allowed value of $q$. Far from the low frequency sector, we can observe a different and irregular behaviour. It happens to some of the frequencies to be strongly almost resonant with multiples of the QB frequency $\omega_{q_{0}}$ (or $\Omega_{q_{0}}$ ). Let us estimate the lower bound $k_{c} q_{0}$ for the existence of such strong almost resonances. It is enough to find the first $k_{c}$ for which

$$
\omega_{k_{c} q_{0}}-\left(k_{c}-1\right) \omega_{q_{0}}=0
$$

holds. Expanding $\omega_{q}$ for small $q$ to third order we find $k_{c} \approx\left(\frac{6 N^{2}}{\pi^{2} q_{0}^{2}}\right)^{\frac{1}{3}}$, or in size-independent quantities $\kappa_{c} \approx\left(\frac{6 \kappa_{0}}{\pi^{2}}\right)^{\frac{1}{3}}$. This value agrees well with the location of the first local minimum in the mentioned figures. The subsequent resonances suggest that the QB profile will show resonant deviations in its tail, exactly at the predicted locations. The sequence $\chi_{j}$ for very small nonlinearities can be well approximated by $\eta_{j}$. Increasing the perturbation, new small denominators may appear and some of the old one can become less relevant due to the QB frequency change.

In Fig. 1(d) the $\chi$ sequence corresponds to the QB in Fig. 1(b). The resonant local minima for $\chi_{j}$ are $j=16$ and $j=21$, which perfectly agree with the two tail peaks of the corresponding QB. Note that the $\eta$ sequence in Fig. 1(c) shows almost the same resonance locations, but the relative strength is different. Also both sequences show 
additional resonance locations, which are not observed in the QB tail. Instead almost all of them are observed in the energy distribution of the corresponding natural packet in Fig. I(a).

The distributions in Fig. 2(a,b) refer to two natural packets localized respectively on $q_{0}=2$ and $q_{0}=3$, while in Fig. 3(a,b) we show the corresponding exact $q_{0}$-breathers. the profiles, including the (much weaker) tail resonances, coincide remarkably. That is due to the weak nonlinearity. The corresponding $\eta$ sequencies in Fig. 2(c,d) and $\chi$ sequencies Fig. B (c,d) also show very good agreement with the really observed locations of tail resonances.

A conjecture which explains the existence of the observed peaks in the tails can be now formulated. The amplitudes of some modes are amplified by very small denominators generated via strong almost exact resonances of the type $\omega_{n q_{0}}^{2}-m^{2} \Omega_{q_{0}}^{2}$. The $\eta$ and $\chi$ sequencies allow to predict possible locations of these superharmonic resonances, however not always the predicted locations are indeed occupied by observed resonances.

Let us now formulate a set of QB properties which have to be studied. First we recall again that the energy distribution of the computed $q$-breathers for $q_{0} \ll N$ shows that their exponential localization is kept throughout the whole $q$-space and is not restricted to the low $q$ mode sector, as anticipated by the approximations used in perturbation theory. Hence, it is an important task to extend the perturbation theory developed in [16] in order to include the large $q$ sector. This would imply to deal with the small divisors without any special Taylor expansion. Moreover, to properly cope with the cascade of modes, one should understand which are the actual modes excited in the $q$ breather, according to the coefficient $B_{i j h}$.

Next, as suggested by several numerical studies, QBs appear to have exponential localization for any $q_{0}$. One can expect that this is a common property of all the Lyapunov orbits in FPU chains. The expected differences, when changing $q_{0}$, could concern the sequence of modes involved and, maybe, details of the exponential decay and stability of QBs.

As shown above, tail resonances in the QB energy distribution appear due to superharmonic almost resonances. These tail resonances are also observed in the evolution of natural packets, and are responsible for the approach to equipartition, pumping energy from the packet core into the tail. An analytical theory would thus allow to understand and describe the ultimate route to equipartition, the holy grail of the FPU problem.

Below we will address some of the above points.

\section{Improved perturbation theory}

In this third part we present a result based on the Poincaré-Lindstedt (PL) perturbation scheme: for a generic $q_{0}$-breather, we prove the existence of a threshold $\mathcal{E}_{q_{0}}^{*}$, scaling with $\kappa_{0}=\frac{q_{0}}{N+1}$ and with the perturbation parameter, such that for the energy density $\mathcal{E}_{q_{0}}<\mathcal{E}_{q_{0}}^{*}$ the 
averaged harmonic energies involved in the QB dynamics are exponentially localized. In the $\alpha$ model, for example, we get for any $n \geq 2$

$$
\begin{aligned}
\left\langle E_{\bar{q}_{n}}\right\rangle & <E_{q_{0}}\left(\omega_{n q_{0}}^{2}+n^{2} \omega_{q_{0}}^{2}\right)\left(\frac{\mu_{n}}{\bar{\mu}_{n-1}}\right)^{2} e^{-(1-n)\left|\ln \gamma_{n}\right|} \\
\gamma_{n} & =\mathcal{C}\left(\alpha \bar{\mu}_{n-1}\right)^{2} \mathcal{E}_{q_{0}}
\end{aligned}
$$

where $\bar{q}_{n}$ is the sequence of the modes excited in the QB, $\left\langle E_{\bar{q}_{n}}\right\rangle$ is the leading averaged harmonic energy

$$
\left\langle E_{\bar{q}_{n}}\right\rangle=\frac{\sigma^{2 n-2}}{\tau} \int_{0}^{\tau} \frac{1}{2}\left[\omega_{n q_{0}}^{2}\left(Q_{\bar{q}_{n}}^{(n-1)}\right)^{2}(t)+\left(P_{\bar{q}_{n}}^{(n-1)}\right)^{2}(t)\right] d t,
$$

and $\mu_{n}, \bar{\mu}_{n}$ are defined as

$$
\mu_{n}:=\frac{1}{\eta_{n}}, \quad \bar{\mu}_{n}=\max _{l=2, \ldots, n}\left\{\mu_{l}\right\} .
$$

This result completes the study on QB localization in Fourier space performed in [16], developing a more general estimate based on the same PL scheme. Among the differences between the two approaches, we first mention the lack of an explicit local approximation for the smallest divisor as in (7): in fact for any $n$ we simply factorize the term $\eta_{n}$ without knowing its value. This allows us to generalize the previous result, once it is clear how to determine $\bar{q}_{n}$. Another difference concerns the harmonic cutoff of the leading solution (6): in order to perform a rigorous approach we have decided to keep all the harmonics in the Fourier expansion of the solution. This leads to the constant factor $\mathcal{C}>1$ in (11), which takes into account both the accumulation of frequencies' products and all the possible non-zero higher harmonics. This affects the threshold $\mathcal{E}_{q_{0}}^{*}$, which is derived from (11) by requesting $\gamma_{n}<1$ :

$$
\mathcal{E}_{q_{0}}^{*}:=\mathcal{C}\left(\frac{1}{\alpha \mu}\right)^{2}, \quad \mu:=\max \left\{\bar{\mu}_{n}\right\} .
$$

We have already mentioned the scaling properties of $\mathcal{E}_{q_{0}}^{*}$, which depends on the wave number $\kappa_{0}$, but not on the actual system size. The main reason is that, when changing both $q_{0}$ and $N+1$ while keeping their ratio fixed, the frequencies involved in the QB evolution are always the same. Then the small denominators also depend only on $\kappa_{0}$ and the same holds for $\mu$. On the other hand, if we fix $q_{0}$ while changing the size $N$ of the system, the smallest possible divisor $\bar{\eta}_{j}$ goes to zero. Consequently the threshold $\mathcal{E}_{q_{0}}^{*}$ vanishes and the QB loses its localization in such a case. In general we are not able to provide an explicit value for $\mu$. In some cases, however, it is possible to make a suitable approximation similar to (7). For example, when $q_{0}$ is close to zero or to $N$, it follows

$$
\mathcal{E}_{q_{0}}^{*} \approx \alpha^{-2} \pi^{4} \kappa_{0}^{4}, \quad \quad \mathcal{E}_{q_{0}}^{*} \approx \alpha^{-2} \pi^{4}\left(1-\kappa_{0}\right)^{4},
$$


for the $\alpha$ model and

$$
\mathcal{E}_{q_{0}}^{*} \approx \beta^{-1} \pi^{2} \kappa_{0}^{2}, \quad \mathcal{E}_{q_{0}}^{*} \approx \beta^{-1} \pi^{2}\left(1-\kappa_{0}\right)^{2},
$$

for the $\beta$ model. In these two specific cases the slope of the exponential decay is given by $\left|\ln \gamma_{2}\right|$ : this value is preserved for almost all the energy distribution, until values of $\mu_{j}$ greater than $\mu_{2}$ appear in the tail. These higher order anomalies are the ones producing resonant tail peaks: the factor $\mu_{n}$ in (11) amplifies those modes whose frequencies are almost resonant with $\omega_{q_{0}}$. This effect is further influenced by $\omega_{n q_{0}}$, which, depending on its value, can weaken or strengthen the amplitudes (see section 3.4 and Fig. 3). An unavoidable limitation is that the sequence $\mu_{j}$ is constant with respect to the perturbation parameters $\alpha$ and $\mathcal{E}_{q_{0}}$. Thus it does not take a possible change of position and intensity of the peaks in the energy distribution into account.

In the following, we will recall the basic ingredients of the PoincaréLindstedt method. Then we present the rules used to predict the cascade of modes excited in any $q_{0}$-breather. Proposition 3.1 can be suitably extended to any homogeneous potential. It is strongly related to some symmetries of the system, as explained in Appendix A and B (see also [32]). Finally we move to the main general statement about localization, performing the proof for $\alpha, \beta$ and a combined $\alpha-\beta$ model. We finish with an application to the high-frequency-mode case $\left|q_{0}-N\right| \ll N$.

\subsection{The Poincaré-Lindstedt method.}

The Poincaré-Lindstedt expansion is a classical perturbation method used to continue a periodic orbit with respect to a small perturbation parameter, when fixing the amplitude (or the energy) of the system. The crucial idea is to expand both the solution and the modified frequency with respect to this parameter: this is essential to kill secular terms which appear in the recursive scheme.

For example, consider directly the $\alpha$-FPU system: we start expanding in $\sigma=\frac{\alpha}{\sqrt{2 N+2}}$ both the normal mode coordinates and the new orbit's frequency

$$
Q_{q}(t)=\sum_{j \geq 0} \sigma^{j} Q_{q}^{(j)}(t), \quad \Omega(\sigma)=\sum_{j \geq 0} \sigma^{j} \omega^{(j)} .
$$

We define a new stretched time variable $\tau=\Omega(\sigma) t$ and we rewrite the equation of motion with respect to the new time derivative $\frac{d}{d \tau}$

$$
\Omega(\sigma)^{2} \frac{d^{2}}{d \tau^{2}} Q_{q}(\tau)+\omega_{q}^{2} Q_{q}(\tau)=-\sigma \sum_{1 \leq l, m \leq N} \omega_{q} \omega_{l} \omega_{m} Q_{l}(\tau) Q_{m}(\tau) B_{q l m} .
$$

If we expand (14) with respect to $\sigma$ we get an infinite sequence of 
equations for every term $Q_{q}$

$$
\begin{aligned}
\sum_{h=0}^{n} \Omega_{h}^{2} \frac{d^{2}}{d \tau^{2}} Q_{q}^{(n-h)} & =-\omega_{q}^{2} Q_{q}^{(n)}- \\
& -\omega_{q} \sum_{l, m=1}^{N} \omega_{l} \omega_{m} B_{q l m} \sum_{h=0}^{n-1} Q_{l}^{(h)} Q_{m}^{(n-1-h)}
\end{aligned}
$$

where

$$
\Omega^{2}(\sigma)=\sum_{h \geq 0} \sigma^{h} \Omega_{h}^{2}, \quad \Omega_{h}^{2}=\sum_{i=0}^{h} \omega^{(i)} \omega^{(h-i)} .
$$

We need solutions of (15) which are $2 \pi$-periodic in $\tau$, so bounded. The initial condition will be chosen so to guarantee this requirement, and the term $\omega^{(j)}$ will be used to prevent the existence of secular terms (unbounded motions) which could appear in the equation of the continued mode.

\subsection{Cascade of modes.}

The QBs computed in Fig. 3 present an interesting feature that was already visible in [16: only a small subset of modes is taking part to the dynamics, the other being essentially at rest. So, for a full description of such solutions, we aim at understanding which mechanism is responsible for this modes' selection. This is also essential in order to get some straightforward result from equation (15). The main rule is that, at any $n$-step of perturbation theory, just one new mode $\bar{q}_{n}$ is excited: this corresponds to the only mode in the interval $[1, N]$ whose frequency equals $2\left|\sin \left(\frac{n q_{0} \pi}{2 N+2}\right)\right|$. The count of $\bar{q}_{n}$ stops when either $n q_{0}$ is a multiple of $N+1$ or when $n q_{0}$ and $(n-1) q_{0}$ have the same frequency (i.e. they are symmetric with respect to one of the two dispersion edges). A more rigorous claim is the following (for a sketch of the proof see Appendix A):

Proposition 3.1 At any $k-1$ perturbation order, only one new mode $\bar{q}_{k}$ with frequency $2\left|\sin \left(\frac{k q_{0} \pi}{2 N+2}\right)\right|$ is excited. This mode reads

$\bar{q}_{k}= \begin{cases}\left(k q_{0}\right) \bmod (2 N+2), & \left(k q_{0}\right) \bmod (2 N+2)<N+1 \\ 2 N+2-\left(k q_{0}\right) \bmod (2 N+2), & \text { otherwise }\end{cases}$

Thus it holds

$$
Q_{\bar{q}_{k}}^{(m)}(t)=0 \quad \forall m<k-1 .
$$

The above proposition exactly predicts which modes are actually excited by the QB's dynamics. These orbits are strictly related to some of those invariant submanifolds whose existence has been proved in 32 using discrete symmetries of the system. However, differently from those arguments, the above proposition provides also an order according to which the modes take part in the evolution.

The final scenario is the following. Take $1 \leq q_{0} \leq N$ and $g_{0}:=$ $\operatorname{gcd}\left(q_{0}, 2 N+2\right)$ (as in Proposition 5.1). For $g_{0}=1$ the submanifold and 
the QB are simply the whole mode space. For $g_{0} \geq 2$ and $\frac{2 N+2}{g_{0}} \notin \mathbb{N}$ the submanifold has lower dimension that the number of modes, at the same time it can not be embedded in a subsystem with fixed boundary conditions. Hence, the QB does not involve all the modes, but is not a rescaled solution of a lower dimensional system. For $g_{0} \geq 2$ and $g_{0}=\operatorname{gcd}\left(N+1, q_{0}\right)$ the submanifold is embedded in a fixed boundary condition subsystem with $\frac{N+1}{g_{0}}$ particles and the QB is the $\frac{q_{0}}{g_{0}}$-mode rescaled solution, constructed as in [25].

\subsection{Exponential localization}

Next we prove the exponential decay of the harmonic energies for a generic QB. This proof makes use of the sequence of small denominators previously denoted as $\eta_{n, m}$, since it naturally arises in the PL method. The Fourier coefficients in the PL scheme, at any new step, are naturally defined via a convolution product. Since $l_{b}^{2}$ is not closed with respect to this product, the choice of a $\|\cdot\|_{l_{b}^{1}} \geq\|\cdot\|_{l_{b}^{2}}$ norm seems natural to control the main physical quantities. It is then useful to recall the following

Lemma 3.1 Let $l_{b}^{1}$ denote the Banach space of absolutely convergent bi-infinite sequences, endowed with the norm $\|a\|_{l_{b}^{1}}:=\sum_{j \in \mathbb{Z}}\left|a_{j}\right|$. Then $l_{b}^{1}$ is a Banach algebra with respect to the convolution product

$$
(a \star b)_{m}=\sum_{i+j=m} a_{i} b_{j}
$$

and

$$
\|a \star b\|_{l_{b}^{1}} \leq\|a\|_{l_{b}^{1}}\|b\|_{l_{b}^{1}}
$$

holds.

\subsubsection{The $\alpha$ model.}

We develop an estimate for the averaged harmonic energies of the lead-

ing terms $Q_{\bar{q}_{k}}^{(k-1)}$, based on the scheme presented at the beginning of this section. By applying Prop. 3.1 and with the same notation for the modes, equation (15) reads

$$
\begin{aligned}
\omega_{q_{0}}^{2} \frac{d^{2}}{d \tau^{2}} Q_{\bar{q}_{k}}^{(k-1)} & =-\omega_{k q_{0}}^{2} Q_{\bar{q}_{k}}^{(k-1)}- \\
& \pm \omega_{k q_{0}} \sum_{l=1}^{k-1} \omega_{l q_{0}} \omega_{(k-l) q_{0}} Q_{\bar{q}_{l}}^{(l-1)} Q_{\bar{q}_{k-l}}^{(k-1-l)}
\end{aligned}
$$

At the first step $(k=2)$

$$
Q_{\bar{q}_{2}}^{(1)}(\tau)= \pm \frac{\omega_{2 q_{0}} \omega_{q_{0}}^{2} A_{q_{0}}^{2}}{2}\left[\frac{1}{\omega_{2 q_{0}}^{2}}+\frac{\cos (2 \tau)}{\omega_{2 q_{0}}^{2}-4 \omega_{q_{0}}^{2}}\right]
$$


We define the quantities

$$
\bar{\eta}_{2, l}:=\left|\omega_{2 q_{0}}^{2}-l^{2} \omega_{q_{0}}^{2}\right|, \quad \eta_{2}:=\min _{l=0, \ldots, 2}\left\{\eta_{2, l}\right\}, \quad \mu_{2}:=\frac{1}{\eta_{2}} .
$$

The solution becomes

$$
Q_{\bar{q}_{2}}^{(1)}(\tau)=\sum_{m=-2}^{2} \hat{C}_{2, m} \exp (i m \tau), \quad \hat{C}_{2, m}=\hat{C}_{2,-m}, \quad C_{2,1}=0,
$$

and the Fourier sequence satisfies

$$
\left\|\hat{C}_{2}\right\|_{l_{b}^{1}} \leq \mu_{2}\left\|\hat{C}_{1}\right\|_{l_{b}^{1}}^{2} c_{2}, \quad c_{2}=\omega_{2 q_{0}} \omega_{q_{0}}^{2} .
$$

Now, we state and prove the main

Theorem 3.1 Define for any $k \geq 2$ the following quantities:

$$
\begin{array}{rlrl}
\bar{\eta}_{k, l} & :=\left|\omega_{k q_{0}}^{2}-l^{2} \omega_{q_{0}}^{2}\right|, & \eta_{k}:=\min _{l=k, k-2, \ldots}\left\{\bar{\eta}_{k, l}\right\}, \\
\mu_{k}:=\frac{1}{\eta_{k}}, & \bar{\mu}_{k}=\max _{l=2, \ldots, k}\left\{\mu_{l}\right\} .
\end{array}
$$

Then, the leading solution $Q_{k q_{0}}^{(k-1)}(\tau)$ reads

$Q_{\bar{q}_{k}}^{(k-1)}(t)=\sum_{m=-k}^{k} \hat{C}_{k, m} \exp (i m \Omega t), \quad \hat{C}_{k, m}=0, m=k-1, \ldots 1-k$,

and there exists a positive constant $a>1$ such that the Fourier sequence $\hat{C}_{k}$ fulfills the following estimate

$$
\left\|\hat{C}_{k}\right\|_{l_{b}^{1}} \leq \mu_{k}\left(\bar{\mu}_{k-1}\right)^{k-2}\left\|\hat{C}_{1}\right\|_{l_{b}^{1}}^{k} c_{k}, \quad \quad c_{k}<\omega_{q_{0}}^{k} a^{k-1} .
$$

Moreover, if $\mathcal{E}_{q_{0}}=\frac{E_{q_{0}}}{N+1}$ represents the energy density of the mode $q_{0}$, there exists a threshold $\mathcal{E}_{q_{0}}^{*}$ such that, for $\mathcal{E}_{q_{0}}<\mathcal{E}_{q_{0}}^{*}$, the leading averaged harmonic energy fulfills

$$
\left\langle E_{\bar{q}_{k}}\right\rangle<E_{q_{0}}\left(\omega_{n q_{0}}^{2}+n^{2} \omega_{q_{0}}^{2}\right)\left(\frac{\mu_{k}}{\bar{\mu}_{k-1}}\right)^{2} e^{-(1-k)\left|\ln \gamma_{k}\right|},
$$

with

$$
\gamma_{k}=2\left(\alpha a \bar{\mu}_{k-1}\right)^{2} \mathcal{E}_{q_{0}} .
$$

proof: By induction. The case $k=2$ has been already proved. Notice that it is not necessary to define $\bar{\mu}_{1}$, since its exponent is 0 . Suppose the statement is true for $k=n-1$ and let us prove it for $k=n$. The time-dependent force is composed of harmonics with frequencies running from 0 to $n$

$$
Q_{\bar{q}_{l}}^{(l-1)}(t) Q_{\bar{q}_{n-l}}^{(n-1-l)}(t)=\sum_{m=-n}^{n}\left(\hat{C}_{l} \star \hat{C}_{n-l}\right)_{m} \exp (i m \Omega t),
$$


with

$$
\left(\hat{C}_{l} \star \hat{C}_{n-l}\right)_{m}=\left(\hat{C}_{l} \star \hat{C}_{n-l}\right)_{-m}
$$

and

$$
\left(\hat{C}_{l} \star \hat{C}_{n-l}\right)_{m}=0, \quad m=n-1, \ldots, 1-n .
$$

From the definition of the force Fourier coefficient

$$
\hat{F}_{n, m}^{(n-1)}=\sum_{l=1}^{n-1} B_{\bar{q}_{n}, \bar{q}_{l}, \bar{q}_{n-l}}\left(\hat{C}_{l} \star \hat{C}_{n-l}\right)_{m} \omega_{l q_{0}} \omega_{(k-l) q_{0}}
$$

we can expand the time dependent force

$$
F_{\bar{q}_{n}}^{(n-1)}(t)=-\omega_{n q_{0}} \sum_{m=-n}^{n} \hat{F}_{n, m}^{(n-1)} \exp (i m \Omega t),
$$

and write the solution as

$$
\begin{aligned}
Q_{\bar{q}_{n}}^{(n-1)}(t) & =-\omega_{n q_{0}} \sum_{m=-n}^{n} \frac{\hat{F}_{n, m}^{(n-1)}}{\omega_{n q_{0}}^{2}-m^{2} \omega_{q_{0}}^{2}} \exp (i m \Omega t)= \\
& =\sum_{m=-n}^{n} \hat{C}_{n, m} \exp (i m \Omega t) .
\end{aligned}
$$

Now we move to the estimate:

$$
\begin{aligned}
\left\|\hat{C}_{n}\right\|_{l_{b}^{1}} & \leq \frac{\omega_{n q_{0}}}{\eta_{n}} \sum_{m=-n}^{n} \frac{\eta_{n}}{\bar{\eta}_{n, m}}\left|\hat{F}_{n, m}^{(n-1)}\right| \leq \\
& \leq \frac{\omega_{n q_{0}}}{\eta_{n}} \sum_{l=1}^{n-1} \omega_{l q_{0}} \omega_{(n-l) q_{0}}\left\|\hat{C}_{l} \star \hat{C}_{n-l}\right\|_{l_{b}^{1}} \leq \\
& \leq \frac{\omega_{n q_{0}}}{\eta_{n}} \sum_{l=1}^{n-1} \omega_{l q_{0}} \omega_{(n-l) q_{0}}\left\|\hat{C}_{l}\right\|_{l_{b}^{1}}\left\|\hat{C}_{n-l}\right\|_{l_{b}^{1}}< \\
& <\frac{\omega_{n q_{0}}}{\eta_{n}}\left\|\hat{C}_{1}\right\|_{l_{b}^{1}}^{n}\left(\bar{\mu}_{n-1}\right)^{n-2} \sum_{l=1}^{n-1} \omega_{l q_{0}} \omega_{(n-l) q_{0}} c_{l} c_{n-l}= \\
& =\mu_{n}\left\|\hat{C}_{1}\right\|_{l_{b}^{1}}^{n}\left(\bar{\mu}_{n-1}\right)^{n-2} c_{n},
\end{aligned}
$$

where we have defined by recurrence

$$
c_{n}:=\omega_{n q_{0}} \sum_{l=1}^{n-1} \omega_{l q_{0}} \omega_{(n-l) q_{0}} c_{l} c_{n-l} .
$$

The values of the first elements $c_{2}, c_{3}, c_{4}, \ldots$ suggest the possibility to collect a factor $\omega_{q_{0}}^{n}$ from any term $c_{n}$ : indeed, if we set $d_{2}=\omega_{2 q_{0}}$ and

$$
d_{n}:=\omega_{n q_{0}}\left(2 d_{n-1} \omega_{(n-1) q_{0}}+\sum_{j=2}^{n-2} \omega_{l q_{0}} \omega_{(n-l) q_{0}} d_{l} d_{n-l}\right)
$$


then $c_{n}=\omega_{q_{0}}^{n} d_{n}$, with $d_{n}<a^{n-1}$. The bound on the amplitudes $c_{n}<\omega_{q_{0}}^{n} a^{n-1}$, which is proved in the Appendix C, allows to estimate the averaged leading terms of the harmonic energies (12)

$$
\left\langle E_{\bar{q}_{n}}\right\rangle=\frac{\sigma^{2 n-2}}{\tau} \int_{0}^{\tau} \frac{1}{2}\left[\omega_{n q_{0}}^{2}\left(Q_{\bar{q}_{n}}^{(n-1)}\right)^{2}(t)+\left(P_{\bar{q}_{n}}^{(n-1)}\right)^{2}(t)\right] d t,
$$

where

$$
P_{\bar{q}_{n}}^{(n-1)}(t)=\sum_{m=-n}^{n} i m \omega_{q_{0}} \hat{C}_{n, m} \exp (i m \Omega t)
$$

is obtained by differentiating $Q_{\bar{q}_{n}}^{(n-1)}(t)$ with respect to time $t$ and recalling that $\Omega=\omega_{q_{0}}+\mathcal{O}(\sigma)$. The period-average yields to

$$
\begin{aligned}
\left\langle E_{\bar{q}_{n}}\right\rangle & =\sigma^{2 n-2} \sum_{|m| \leq n} \frac{1}{2}\left(\omega_{n q_{0}}^{2}+m^{2} \omega_{q_{0}}^{2}\right)\left|\hat{C}_{n, m}\right|^{2}< \\
& <\sigma^{2 n-2} \frac{1}{2}\left(\omega_{n q_{0}}^{2}+n^{2} \omega_{q_{0}}^{2}\right) \sum_{|m| \leq n}\left|\hat{C}_{n, m}\right|^{2} \leq \\
& \leq \sigma^{2 n-2} \frac{1}{2}\left(\omega_{n q_{0}}^{2}+n^{2} \omega_{q_{0}}^{2}\right)\left\|\hat{C}_{n}\right\|_{l_{b}^{1}}^{2} .
\end{aligned}
$$

By means of the previous estimate on $\left\|\hat{C}_{n}\right\|_{l_{b}^{1}}$ we get

$$
E_{\bar{q}_{n}}^{(n-1)} \leq E_{q_{0}}\left(\omega_{n q_{0}}^{2}+n^{2} \omega_{q_{0}}^{2}\right)\left(\frac{\mu_{n}}{\bar{\mu}_{n-1}}\right)^{2} e^{-(1-n)\left|\ln \gamma_{n}\right|}
$$

which is an exponential decay until

$$
\gamma_{n}=2\left(\alpha a \bar{\mu}_{n-1}\right)^{2} \mathcal{E}_{q_{0}}<1, \quad \forall n=1, \ldots, N-1 .
$$

If we define $\mu=\max \bar{\mu}_{n}$, the condition

$$
\gamma<1 \quad \Leftrightarrow \quad \mathcal{E}_{q_{0}}<\mathcal{E}_{q_{0}}^{*}:=2(a \alpha \mu)^{-2}
$$

repesents the $q_{0}$ specific energy's threshold for the exponential decay.

\subsubsection{The $\beta$ model.}

For the $\beta$-model we give the main ideas how to extend the perturbation estimates performed for the $\alpha$ model. The quartic part of the potential can be rewritten as

$$
\frac{\beta}{4} \sum_{j=0}^{N}\left(x_{j+1}-x_{j}\right)^{4}=\frac{\beta}{8(N+1)} \sum_{1 \leq i, j, h, k \leq N} \omega_{i} \omega_{j} \omega_{h} \omega_{k} Q_{i} Q_{j} Q_{h} Q_{k} B_{i j h k},
$$


where we set $B_{i j h k}=\sum_{\sigma_{1,2,3}= \pm 1} \Delta_{i+\sigma_{1} j+\sigma_{2} h+\sigma_{3} k}$, with

$$
\Delta_{i \pm j \pm h \pm k}=\left\{\begin{array}{cc}
1, & i \pm j \pm h \pm k=0 \\
-1, & i \pm j \pm h \pm k= \pm 2(N+1) . \\
0, & \text { otherwise }
\end{array}\right.
$$

The equation of motion for a normal mode then reads

$$
\ddot{Q_{q}}+\omega_{q}^{2} Q_{q}=-\frac{\beta}{2(N+1)} \sum_{1 \leq i, j, h \leq N} \omega_{q} \omega_{i} \omega_{j} \omega_{h} Q_{i} Q_{j} Q_{h} B_{q i j h} .
$$

The small parameter used in the Taylor expansion is $\sigma=\frac{\beta}{2 N+2}$. With this choice, the equation at every order $s$ becomes

$$
\begin{aligned}
\sum_{r=0}^{s} \Omega_{r}^{2} \frac{d^{2}}{d \tau^{2}} Q_{q}^{(s-r)} & =-\omega_{q}^{2} Q_{q}^{(s)}- \\
& -\omega_{q} \sum_{i, j, h=1}^{N} \omega_{i} \omega_{j} \omega_{h} B_{q i j h} \sum_{l+m+n=s-1} Q_{i}^{(l)} Q_{j}^{(m)} Q_{h}^{(n)} .
\end{aligned}
$$

Following both [16] and Proposition [3.1] we can prove

Proposition 3.2 At any $s-1$ perturbation order a new mode $\bar{q}_{s}$ with frequency $2\left|\sin \left(\frac{(2 s-1) q_{0} \pi}{2 N+2}\right)\right|$ is excited:

$$
\bar{q}_{s}=\left\{\begin{array}{l}
(2 s-1) q_{0} \bmod (2 N+2), \quad(2 s-1) q_{0} \bmod (2 N+2)<N+1 \\
2 N+2-(2 s-1) q_{0} \bmod (2 N+2), \quad \text { otherwise }
\end{array}\right.
$$

Thus

$$
Q_{\bar{q}_{s}}^{(m)}(t)=0 \quad \forall m<s-1 .
$$

Consider $s=2$ : the solution of

$$
\omega_{q_{0}}^{2} \frac{d^{2}}{d \tau^{2}} Q_{\bar{q}_{2}}^{(1)}+\omega_{3 q_{0}}^{2} Q_{\bar{q}_{2}}^{(1)}= \pm \omega_{3 q_{0}} \omega_{q_{0}}^{3}\left(Q_{q_{0}}^{(0)}\right)^{3}
$$

is given by

$$
Q_{\bar{q}_{2}}^{(1)}(\tau)= \pm \omega_{3 q_{0}} \omega_{q_{0}}^{3} A_{q_{0}}^{3}\left[\frac{3}{4} \frac{\cos (\tau)}{\omega_{3 q_{0}}^{2}-\omega_{q_{0}}^{2}}+\frac{1}{4} \frac{\cos (3 \tau)}{\omega_{3 q_{0}}^{2}-9 \omega_{q_{0}}^{2}}\right],
$$

or, after expanding cosine terms

$$
Q_{\bar{q}_{2}}^{(1)}(\tau)=\sum_{m=-3}^{3} \hat{C}_{2, m} \exp (i m \Omega t), \quad \hat{C}_{2,2 m}=0, \quad \hat{C}_{2, m}=\hat{C}_{2,-m} .
$$

We define the following quantities

$$
\eta_{2, l}:=\left|\omega_{3 q_{0}}^{2}-l^{2} \omega_{q_{0}}^{2}\right|, \quad \overline{\eta_{2}}:=\min _{l=1,3}\left\{\eta_{2, l}\right\}, \quad \mu_{2}:=\frac{1}{\overline{\eta_{2}}},
$$


then

$$
\left\|\hat{C}_{2}\right\|_{l_{b}^{1}} \leq \mu_{2}\left\|\hat{C}_{1}\right\|_{l_{b}^{1}}^{3} c_{2}, \quad c_{2}=\omega_{3 q_{0}} \omega_{q_{0}}^{3} .
$$

Theorem 3.2 Define for any $s \geq 2$ the following quantities:

$$
\begin{array}{rlrl}
\bar{\eta}_{s, l} & :=\left|\omega_{(2 s-1) q_{0}}^{2}-l^{2} \omega_{q_{0}}^{2}\right|, & \eta_{s}:=\min _{l=1,3, \ldots, 2 s-1}\left\{\eta_{s, l}\right\}, \\
\mu_{s}:=\frac{1}{\eta_{s}}, & \bar{\mu}_{s}=\max _{l=2, \ldots, s}\left\{\mu_{s}\right\} .
\end{array}
$$

Then, the leading solution $Q_{\bar{q}_{s}}^{(s-1)}(t)$ reads

$$
Q_{\bar{q}_{s}}^{(s-1)}(t)=\sum_{m=-2 s+1}^{2 s-1} \hat{C}_{s, m} \exp (i m \Omega t), \quad \hat{C}_{s, 2 m}=0,
$$

and there exists a positive constant $a>1$ such that the Fourier sequence $\hat{C}_{s}$ fulfills the following estimate

$$
\left\|\hat{C}_{s}\right\|_{l_{b}^{1}} \leq \mu_{s}\left(\bar{\mu}_{s-1}\right)^{s-2}\left\|\hat{C}_{1}\right\|_{l_{b}^{1}}^{2 s-1} c_{s}, \quad c_{s}<\omega_{q_{0}}^{2 s-1} a^{s-1} .
$$

Moreover, if $\mathcal{E}_{q_{0}}=\frac{E_{q_{0}}}{N+1}$ represents the energy density of the mode $q_{0}$, there exists a threshold $\mathcal{E}_{q_{0}}^{*}$ such that, for $\mathcal{E}_{q_{0}}<\mathcal{E}_{q_{0}}^{*}$

$$
\left\langle E_{\bar{q}_{s}}\right\rangle<E_{q_{0}}\left(\omega_{(2 s-1) q_{0}}^{2}+(2 s-1)^{2} \omega_{q_{0}}^{2}\right)\left(\frac{\mu_{s}}{\bar{\mu}_{s-1}}\right)^{2} e^{-2(1-s)\left|\ln \gamma_{s}\right|},
$$

with

$$
\gamma_{s}=\beta a \bar{\mu}_{s-1} \mathcal{E}_{q_{0}} .
$$

proof:

We give some hints how to modify the proof of Theorem 3.1 The nonlinearity in the equation of motion for $Q_{\bar{q}_{s}}^{(s-1)}$ is

$$
\omega_{(2 s-1) q_{0}} \sum_{l+m+n=s+1} \omega_{(2 l-1) q_{0}} \omega_{(2 m-1) q_{0}} \omega_{(2 n-1) q_{0}} Q_{\bar{q}_{l}}^{(l-1)} Q_{\bar{q}_{m}}^{(m-1)} Q_{\bar{q}_{n}}^{(n-1)},
$$

where $l, m, n \geq 1$. We can define the force Fourier coefficient

$$
\hat{F}_{s, h}^{(s-1)}=\sum_{l+m+n=s+1} B_{\bar{q}_{s}, \bar{q}_{l}, \bar{q}_{m} \bar{q}_{n}}\left(\hat{C}_{l} \star \hat{C}_{m} \star \hat{C}_{n}\right)_{h} \omega_{(2 l-1) q_{0}} \omega_{(2 m-1) q_{0}} \omega_{(2 n-1) q_{0}}
$$

and obtain the time dependent force

$$
F_{\bar{q}_{s}}^{(s-1)}(t)=-\omega_{(2 s-1) q_{0}} \sum_{h=-2 s+1}^{2 s-1} \hat{F}_{s, h}^{(s-1)} \exp (i h \Omega t) .
$$

This, as before, yields the solution

$$
Q_{\bar{q}_{s}}^{(s-1)}(t)=-\omega_{(2 s-1) q_{0}} \sum_{h=-2 s+1}^{2 s-1} \frac{\hat{F}_{s, h}^{(s-1)}}{\omega_{(2 s-1) q_{0}}^{2}-h^{2} \omega_{q_{0}}^{2}} \exp (i h \Omega t)=
$$




$$
=\sum_{h=-2 s+1}^{2 s-1} \hat{C}_{s, h} \exp (i h \Omega t) .
$$

The conclusion of the proof follows Theorem 3.1

\subsubsection{The $\alpha-\beta$ model with $\beta=\frac{2}{3} \alpha^{2}$.}

We finally consider the $\alpha-\beta$ model, under the restriction $\beta=\frac{2}{3} \alpha^{2}$. This dependence is due to the need of expanding the solution with respect to one perturbative parameter only. Moreover, with this choice the potential coincides with the fourth truncation order of the Toda potential and the scheme performed can be adapted to any order. reads

In this case, defining $\sigma=\frac{\alpha}{\sqrt{2 N+2}}$, the equation at any order $s-1$

$$
\begin{aligned}
\sum_{r=0}^{s-1} \Omega_{r}^{2} \frac{d^{2}}{d \tau^{2}} Q_{q}^{(s-1-r)} & =-\omega_{q}^{2} Q_{q}^{(s-1)}-\omega_{q} \sum_{i, j=1}^{N} \omega_{i} \omega_{j} B_{q i j} \sum_{l+m=s-2} Q_{i}^{(l)} Q_{j}^{(m)}- \\
& -\frac{2}{3} \omega_{q} \sum_{i, j, h=1}^{N} \omega_{i} \omega_{j} \omega_{h} C_{q i j h} \sum_{l+m+n=s-3} Q_{i}^{(l)} Q_{j}^{(m)} Q_{h}^{(n)} .
\end{aligned}
$$

As in the $\alpha$ model, the set of modes involved in the dynamics is selected according to Proposition 3.1 (see again Appendix A)

Proposition 3.3 At any $k-1$ perturbation order, only one new mode $\bar{q}_{k}$ with frequency $2\left|\sin \left(\frac{k q_{0} \pi}{2 N+2}\right)\right|$ is excited:

$\bar{q}_{k}= \begin{cases}\left(k q_{0}\right) \bmod (2 N+2), & \left(k q_{0}\right) \bmod (2 N+2)<N+1 \\ 2 N+2-\left(k q_{0}\right) \bmod (2 N+2), & \text { otherwise }\end{cases}$

Thus

$$
Q_{\bar{q}_{k}}^{(m)}(t)=0 \quad \forall m<k-1
$$

The exponential decay is similar to the one obtained for the $\alpha$ model, except for the coefficient $c_{s}$ which is responsible for the accumulation of frequencies:

Theorem 3.3 Define for any $s \geq 2$ the following quantities:

$$
\begin{array}{rlrl}
\bar{\eta}_{s, l} & :=\left|\omega_{s q_{0}}^{2}-l^{2} \Omega_{q_{0}}^{2}\right|, & \eta_{s}:=\min _{l=s, s-2, \ldots}\left\{\eta_{s, l}\right\}, \\
\mu_{s}:=\frac{1}{\eta_{s}}, & \bar{\mu}_{s}=\max _{l=2, \ldots, s}\left\{\mu_{l}\right\} .
\end{array}
$$

Then any solution $Q_{\bar{q}_{s}}^{(s-1)}(t)$ reads

$Q_{\bar{q}_{s}}^{(s-1)}(t)=\sum_{m=-s}^{s} \hat{C}_{s, m} \exp (i m \Omega t), \quad \hat{C}_{s, m}=0, m=s-1, \ldots, 1-s$ 
and there exists a positive constant $a>1$ such that the Fourier sequence $\hat{C}_{s}$ fulfills the following estimate

$$
\left\|\hat{C}_{s}\right\|_{l_{b}^{1}} \leq \mu_{s}\left(\bar{\mu}_{s-1}\right)^{s-2}\left\|\hat{C}_{1}\right\|_{l_{b}^{1}}^{s} c_{s}, \quad c_{s}<\omega_{q_{0}}^{s} a^{s-1} .
$$

Moreover, if $\mathcal{E}_{q_{0}}=\frac{E_{q_{0}}}{N+1}$ represents the energy density of the mode $q_{0}$, there exists a threshold $\mathcal{E}_{q_{0}}^{*}$ such that, for $\mathcal{E}_{q_{0}}<\mathcal{E}_{q_{0}}^{*}$, holds true

$$
\left\langle E_{\bar{q}_{s}}\right\rangle<E_{q_{0}}\left(\omega_{s q_{0}}^{2}+s^{2} \omega_{q_{0}}^{2}\right)\left(\frac{\mu_{s}}{\bar{\mu}_{s-1}}\right)^{2} e^{-(1-s)\left|\ln \gamma_{s}\right|},
$$

with

$$
\gamma_{s}=2\left(\alpha a \bar{\mu}_{s-1}\right)^{2} \mathcal{E}_{q_{0}} .
$$

proof: By induction we consider the step $\bar{q}_{s}$. The quadratic and cubic force are composed of terms like

$$
\begin{aligned}
Q_{\bar{q}_{l}}^{(l-1)} Q_{\bar{q}_{m}}^{(m-1)} & =\sum_{r=-s}^{s}\left(\hat{C}_{l} \star \hat{C}_{m}\right)_{r} \exp (i r \Omega t), \\
Q_{\bar{q}_{l}}^{(l-1)} Q_{\bar{q}_{m}}^{(m-1)} Q_{\bar{q}_{n}}^{(n-1)} & =\sum_{r=-s}^{s}\left(\hat{C}_{l} \star \hat{C}_{m} \star \hat{C}_{n}\right)_{r} \exp (i r \Omega t),
\end{aligned}
$$

with respectively $l+m=s$ and $l+m+n=s$; observe that

$$
\left(\hat{C}_{l} \star \hat{C}_{m}\right)_{r}=\left(\hat{C}_{l} \star \hat{C}_{m} \star \hat{C}_{n}\right)_{r}=0, \quad r=s-1, s-3, \ldots, 1-s .
$$

Since in both the cases the harmonics run from $-s$ to $s$, it is possible to define the force Fourier coefficient as

$$
\begin{aligned}
\hat{F}_{s, r}^{(s-1)} & =\sum_{l=1}^{s-1}\left(\hat{C}_{l} \star \hat{C}_{s-l}\right)_{r} \omega_{l q_{0}} \omega_{(s-l) q_{0}}+ \\
& +\sum_{l=1}^{s-2}\left(\hat{C}_{l} \star \hat{C}_{m} \star \hat{C}_{s-l-m}\right)_{r} \omega_{l q_{0}} \omega_{m q_{0}} \omega_{(s-l-m) q_{0}}
\end{aligned}
$$

and expand the time dependent force

$$
F_{\bar{q}_{s}}^{(s-1)}=-\omega_{s q_{0}} \sum_{r=-s}^{s} \hat{F}_{s, r}^{(s-1)} \exp (i r \Omega t)
$$

which leads to the solution

$$
Q_{\bar{q}_{s}}^{(s-1)}=-\omega_{s q_{0}} \sum_{r=-s}^{s} \frac{\hat{F}_{s, r}^{(s-1)}}{\omega_{s q_{0}}^{2}-r^{2} \Omega_{q_{0}}^{2}} \exp (i r \Omega t)=\sum_{r=-s}^{s} \hat{C}_{s, r} \exp (i r \Omega t) .
$$

It is remarkable that the both force contributions generate the same small denominators. The proof can be then easily finished, using a new 

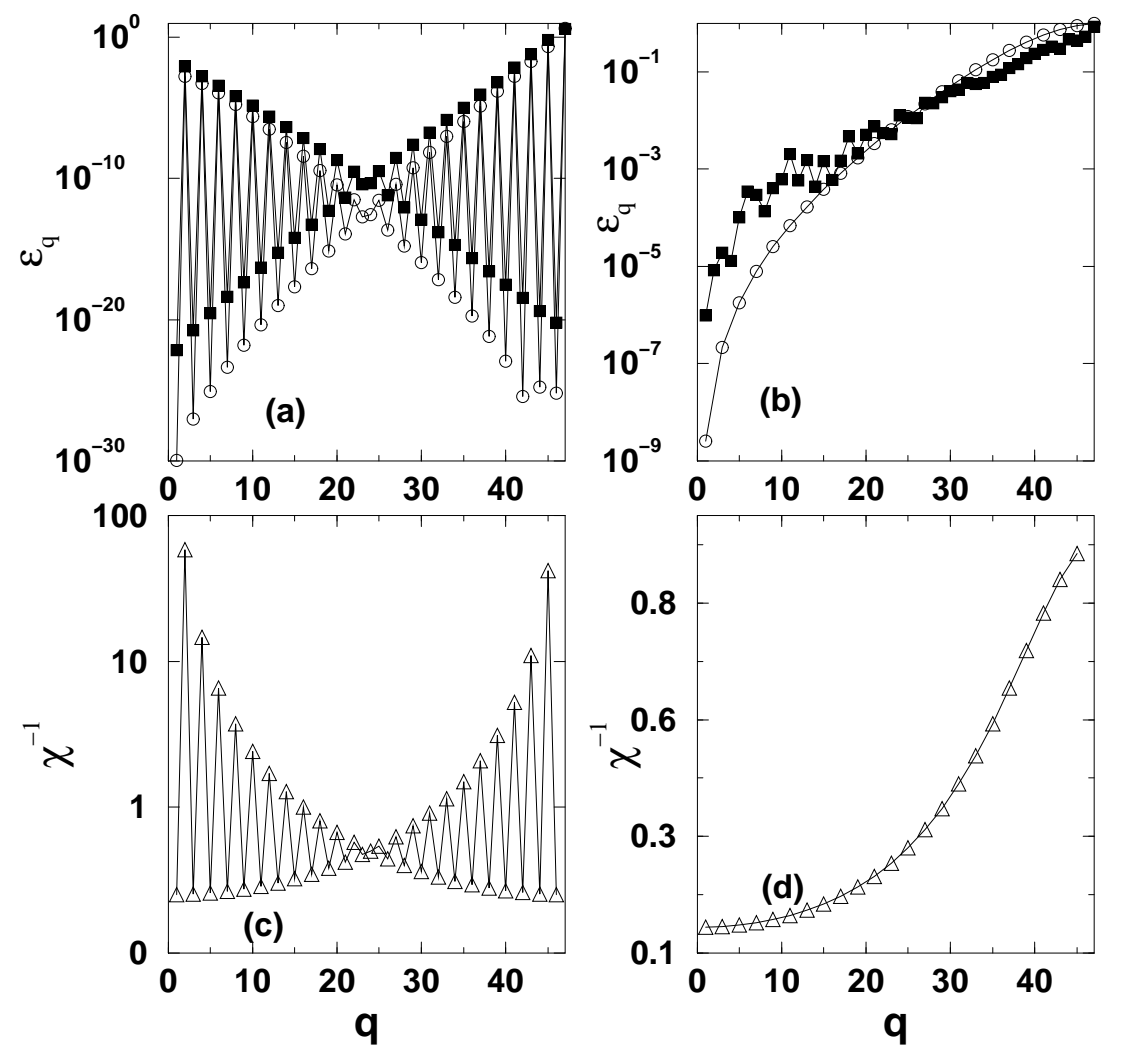

Figure 4: Parameters: $N=47, \mathcal{E}=0.1$. (a): Energy of normal modes in a QB (open symbols) and a natural packet (filled symbols, waiting time $10^{5}$ ) for $q_{0}=47$ and $\alpha=0.25$. (b): Similar to (a), but for $\beta=0.25$. (c): sequence $\chi_{j}^{-1}$ which corresponds to case (a). (d): sequence $\chi_{j}^{-1}$ which corresponds to (b).

definition for $c_{s}$

$$
c_{s}:=\omega_{s q_{0}}\left(\sum_{l=1}^{s-1} \omega_{l q_{0}} \omega_{(s-l) q_{0}} c_{l} c_{s-l}+\sum_{l=1}^{s-2} \omega_{l q_{0}} \omega_{m q_{0}} \omega_{(s-l-m) q_{0}} c_{l} c_{m} c_{s-l-m}\right) .
$$

\subsubsection{Application: the high mode case.}

We consider the case when $q_{0}$ corresponds to a large frequency, i.e. $N-q_{0} \ll 1$. As for a low frequency initial excitation, also this special case is related to the equipartition issue. We recall, for example, that in 35 the energy flow from initially high-frequency-mode excitation to low modes was studied, restricting to a $\beta$-FPU model. The claim was 
that, due to the same mechanism of resonance overlapping described in [10], the equipartition time $T_{e q}$ scales as a power law with the inverse of the energy density, $T_{e q} \sim \mathcal{E}^{-2}$. However, the authors didn't exclude also the possibility that, below a small energy density threshold, the dependence $T_{e q}(\mathcal{E})$ could be even slower than any power law. The existence of exponentially long waiting times to equipartition, scaling with the inverse of the energy density, and the existence of two different time scales for the energy spreading among modes, have been later numerically confirmed in 28].

While the impact of QBs on these observations is essentially the same as for the low frequency case, a new element in the structure of the QB itself arises. For energy densities small enough, it is still evident an exponential decay of harmonic energies starting from the initially excited mode. However, a remarkable difference with the lowmode case exists and it is related to the effect of the $\alpha$ term in the potential: if in the pure $\beta$ model the energy is mainly shared among high frequency modes, in the $\alpha$ case the energy distribution involves, from the very beginning of the evolution, also low frequency modes. We compute QBs with high frequencies, and plot their energy distributions in Fig. 4 The panels (a) and (b) of Fig. 4 show two $q$-breathers with the same largest $q_{0}$, for the $\alpha$ and $\beta$ model, respectively. We also plot the energy distribution of the corresponding natural packets. We again recover that both distributions are remarkably similar. Note that for the $\beta$-model the modes decay monotonously with decreasing mode number. However for the $\alpha$-model a strong excitation of low frequency modes is observed as well.

The two lower panels (c) and (d) in Fig. 4 represent the $\chi_{j}$ sequences corresponding to the two QB periodic orbits. Since in the forthcoming part we want to obtain, from the general definition of $\mathcal{E}^{*}$ in Theorem 3.1 an approximated but explicit dependence of the threshold energy density on $\kappa_{0}:=\frac{q_{0}}{N+1}$, we will actually consider the sequence $\eta_{j}$ instead, since $\eta_{j}$ is still very close to $\chi_{j}$. We deal with the two initial small divisors $\eta_{2}$ and $\eta_{3}$, arising respectively from the first and second PL step. In the first step, the low mode $2 N+2-2 q_{0}$ is coupled to $q_{0}$, but the forcing term $Q_{q_{0}}^{(0)}(t)$ includes only even harmonics, thus generating in the solution a small denominator $\frac{1}{\omega_{2 q_{0}}^{2}}$. We expand $\omega_{2 q_{0}}$ around $\pi$ and obtain the approximation

$$
\omega_{2 q_{0}}^{2}=4 \sin ^{2}\left(\pi\left(1-\kappa_{0}\right)\right) \approx 4 \pi^{2}\left(1-\kappa_{0}\right)^{2} .
$$

In the second step, instead, the mode $3 q_{0}-2 N-2$ is excited. The odd harmonics of the time periodic force generate the small denominator $\frac{1}{\omega_{3 q_{0}}^{2}-\omega_{q_{0}}^{2}}$. Since both $\omega_{3 q_{0}}$ and $\omega_{q_{0}}$ are very close to 2 , their difference is expected to be quadratic in $\left(1-\kappa_{0}\right)$. We expand $\omega_{3 q_{0}}$ in a neighbourhood of $\frac{3}{2} \pi$

$\omega_{3 q_{0}}=2 \sin \left(\frac{3}{2} \pi \kappa_{0}\right)=2 \cos \left(\frac{3}{2} \pi-\frac{3}{2} \pi \kappa_{0}\right)=2-\frac{9}{4} \pi^{2}\left(1-\kappa_{0}\right)^{2}+$ h.o.t. 
and $\omega_{q_{0}}$ around $\frac{1}{2} \pi$

$$
\omega_{q_{0}}=2 \sin \left(\frac{1}{2} \pi \kappa_{0}\right)=2 \cos \left(\frac{1}{2} \pi-\frac{1}{2} \pi \kappa_{0}\right)=2-\frac{1}{4} \pi^{2}\left(1-\kappa_{0}\right)^{2}+\text { h.o.t. }
$$

thus deducing

$$
\left|\omega_{3 q_{0}}^{2}-\omega_{q_{0}}^{2}\right| \approx 8 \pi^{2}\left(1-\kappa_{0}\right)^{2} .
$$

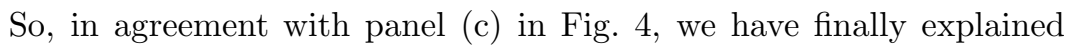
the relationship $\eta_{2}>\eta_{3}$. Thus we may expect $\left\langle E_{\bar{q}_{2}}\right\rangle>\left\langle E_{\bar{q}_{3}}\right\rangle$. To compare panel (c) with panel (a) in Fig. 目 we recall that the averaged harmonic energies $\left\langle E_{\bar{q}_{s}}\right\rangle$ are also corrected by the coefficients $\left(\omega_{s q_{0}}^{2}+\right.$ $\left.s^{2} \omega_{q_{0}}^{2}\right)$. Finally, from the previous local approximation of strong nearby resonances, one can deduce

$$
\mathcal{E}_{q_{0}}^{*} \approx \alpha^{-2} \pi^{4}\left(1-\kappa_{0}\right)^{4} .
$$

It is straightforward to deal with the $\beta$ case and obtain from Theorem 3.2

$$
\mathcal{E}_{q_{0}}^{*} \approx \beta^{-1} \pi^{2}\left(1-\kappa_{0}\right)^{2} .
$$

\section{Conclusions.}

We have studied the energy localization of a generic Lyapunov orbit, called $q$-breather, in the pure $\alpha$ and $\beta$ FPU model. We have proved, thus generalizing a result from [16, that any $q_{0}$-breather exhibits an exponential localization in the Fourier space if the $q_{0}$-mode energy density $\mathcal{E}_{q_{0}}$ is below a threshold $\mathcal{E}_{q_{0}}^{*}$ which depends only on the coupling parameter (either $\alpha$ or $\beta$ ) and on the wave number $\kappa_{0}=\frac{q_{0}}{N+1}$. The specific value of $\mathcal{E}_{q_{0}}^{*}$ suggests that a $\beta$-QB will delocalize for higher energies. Moreover, the exponential decay of amplitudes in a QB runs through a subset of normal modes: we revealed the algebraic rule which selects the modes involved in the QB dynamics, thus explaining their order in the perturbation decay. This rule is strictly linked to the discrete symmetries of the system. Any QB lies on one of those invariant submanifolds described, for example, in 29] and 32].

The possibility to evaluate the Poincaré-Lindstedt (PL) perturbation scheme at any order, allows a qualitative explanation of the previously observed resonant peaks in the tail of the energy distribution. In the numerical experiments on natural packets with initial low frequency modes, we notice anomalies in the tail of the energy distribution. They pump energy from the natural packet core into the tail. We think that this effect is of central relevance to the pathway to equipartition. Such anomalies are also characteristic of any QB, even if in the low fequency mode simulations (see Fig. 1 and Fig. 3) they are more visible. While these peaks have been plotted (but not discussed) already in [16, we have developed a scheme which is capable of explaining the positions of these peaks. We have shown that ther is a direct connection between the position and intensity of these peaks and nonlinear resonances in- 
volving the QB's frequency $\Omega_{q_{0}}$

$$
\bar{\chi}_{n, m}=\left|\omega_{n q_{0}}^{2}-m^{2} \Omega_{q_{0}}^{2}\right| .
$$

In the limit of very small perturbations, the sequence (28) reduces to

$$
\bar{\eta}_{n, m}=\left|\omega_{n q_{0}}^{2}-m^{2} \omega_{q_{0}}^{2}\right|,
$$

which is the one arising in the PL scheme. We have used the latter one for a rigorous analytical approach. We still lack a suitable Fourier expansion which allows to implement recursive estimates including the true small divisors (28). Nevertheless it is possible, restricting to low and high frequencies, to improve the estimate of Theorems 3.1 and 3.2 with a modified PL approach, thus discovering that $\beta$ QB thresholds are larger than $\alpha$ ones. Finally, we have also extended the oneparameter PL perturbation scheme to a special $\alpha-\beta$ model, with $\beta=\frac{2}{3} \alpha^{2}$. Thus the PL method developed, although fitting the numerical simulations only for perturbations small enough, gives a rigorous analytical way to describe localization in Fourier space for any homogeneous FPU-like potential and any truncated Toda potential.

We did not consider the linear stability fo QBs here. The reason is that we focussed on the exponential localization of QBs. This property we believe is not depending on whether the QB is stable or not. Let us explain this point. If a QB is exponentially localized, the core can not be in strong resonance with the tail modes - otherwise the QB would not localize. Thus almost any instability which a QB may encounter on the path of its continuation, will affect the dynamics of the modes in the core of the excitation. Indeed, it has been observed in [10, that such an instability triggers weak chaos among the core modes, leaving the localization intact. The threshold to this weak chaos state was correctly identified as an instability threshold of QBs [16]. Thus, given a QB is localized, its stability or instability will not change the fact that nearby trajectories (natural packets) will stay for long times in that phase space part. 


\section{Appendix.}

\subsection{Appendix A.}

We give here a sketch of the proof of Prop. 3.1 We have decided to omit the proofs of Prop. 3.2 and Prop. 3.3 since, a part from small variations, they are based on the same ideas.

Proof of Proposition [3.1 We give an inductive proof. For $k=2$ the statement is true. Suppose, by inductive hypothesis, that it is also true until $k=n-1$. Let us introduce here the following notation for the modes excited $q_{0}=\bar{q}_{1}, \bar{q}_{2}, \ldots, \bar{q}_{n-1}$ and the repeated dispersion law $F(x):=2\left|\sin \left(\frac{x \pi}{2 N+2}\right)\right|$; the frequencies are respectively

$$
F\left(q_{0}\right), F\left(2 q_{0}\right), \ldots, F\left((n-1) q_{0}\right) .
$$

By the inductive hypothesis, any mode $\bar{q}_{j}$ is obtained from $j q_{0}$ through

$$
\bar{q}_{j}= \begin{cases}J(2 N+2)-j q_{0}, & F^{\prime}\left(j q_{0}\right)>0 \\ j q_{0}-J(2 N+2), & F^{\prime}\left(j q_{0}\right)<0\end{cases}
$$

for some $J$ related to $j q_{0}$. From (15) we know that the equations of motion at the next order include products like

$$
\begin{aligned}
& B_{q \bar{q}_{l} \bar{q}_{m}} Q_{\bar{q}_{l}}^{(0)} Q_{\bar{q}_{m}}^{(n-2)} \\
& B_{q \bar{q}_{l}} \bar{q}_{m} Q_{\bar{q}_{l}}^{(1)} Q_{\bar{q}_{m}}^{(n-3)}
\end{aligned}
$$

and, among them, the only ones giving a new frequency are

$$
\begin{aligned}
& Q_{\bar{q}_{1}}^{(0)} Q_{\bar{q}_{n-1}}^{(n-2)} \\
& Q_{\bar{q}_{2}}^{(1)} Q_{\bar{q}_{n-2}}^{(n-3)}
\end{aligned}
$$

Indeed, by induction $Q_{\bar{q}_{m}}^{(n-h-1)}=0$ for $m>n-h$, and $q \pm \bar{q}_{l} \pm \bar{q}_{m}=$ $0 \bmod (2 N+2)$ is a necessary condition for $B_{q \bar{q}_{l} \bar{q}_{m}} \neq 0$. We claim that the equations giving the new $q$ modes

$$
\begin{aligned}
& q \pm \bar{q}_{1} \pm \bar{q}_{n-1}=0,2 N+2, \\
& q \pm \bar{q}_{2} \pm \bar{q}_{n-2}=0,2 N+2,
\end{aligned}
$$


all admit a common solution, which reads

$$
\bar{q}_{n}= \begin{cases}\bar{N}(2 N+2)-n q_{0}, & F^{\prime}\left(n q_{0}\right)>0 \\ n q_{0}-\bar{N}(2 N+2), & F^{\prime}\left(n q_{0}\right)<0\end{cases}
$$

As an example we consider just the second equation in the previous list. Let us assume that

$$
\begin{aligned}
\bar{q}_{2} & =l(2 N+2)-2 q_{0}, \\
\bar{q}_{n-2} & =L(2 N+2)-(n-2) q_{0}
\end{aligned}
$$

then the possible combinations are

$$
\begin{aligned}
\bar{q}_{2}+\bar{q}_{n-2} & =(L+l)(2 N+2)-n q_{0}, \\
-\bar{q}_{2}-\bar{q}_{n-2} & =-(L+l)(2 N+2)+n q_{0}, \\
\bar{q}_{2}-\bar{q}_{n-2} & =(l-L)(2 N+2)+n q_{0}-4 q_{0}, \\
-\bar{q}_{2}+\bar{q}_{n-2} & =(L-l)(2 N+2)-n q_{0}+4 q_{0} .
\end{aligned}
$$

The last couple give the already included mode $\bar{q}_{n-4}$, so we focus on the first couple, giving the four equations

$$
\begin{aligned}
q+\bar{q}_{2}+\bar{q}_{n-2}=0, & q-\bar{q}_{2}-\bar{q}_{n-2}=0 \\
q+\bar{q}_{2}+\bar{q}_{n-2} & =2 N+2, \quad q-\bar{q}_{2}-\bar{q}_{n-2}=2 N+2
\end{aligned}
$$

notice that the first and the last above equations don't admit a solution $q \in[1, N]$, so the only solutions are

$$
\begin{aligned}
& q=2 N+2-\bar{q}_{2}-\bar{q}_{n-2}=n q_{0}-(L+l-1)(2 N+2) \\
& q=\bar{q}_{2}+\bar{q}_{n-2}=(L+l)(2 N+2)-n q_{0}
\end{aligned}
$$

when respectively $\bar{q}_{2}+\bar{q}_{n-2}>N+1$ and $\bar{q}_{2}+\bar{q}_{n-2}<N+1$; they both correspond to the same frequency $2\left|\sin \left(\frac{n q_{0} \pi}{2 N+2}\right)\right|$.

\subsection{Appendix B.}

Let

$$
\begin{aligned}
R: & :\left(Q_{j}, P_{j}\right) \mapsto\left(Q_{j+1}, P_{j+1}\right) \\
S \quad: \quad\left(Q_{j}, P_{j}\right) & \mapsto\left(-Q_{2 N+2-j},-P_{2 N+2-j}\right)
\end{aligned}
$$

the discrete symmetries of the FPU system and, for a generic symmetry $G$ on a manifold $\mathcal{M}$,

$$
F i x(G):=\{x \in \mathcal{M} \mid G(x)=x\}
$$


It is possible to prove the following

Proposition 5.1 Let $q_{0}$ be a mode to be continued, $g_{0}:=\operatorname{gcd}(2 N+$ $\left.2, q_{0}\right)$ and $\kappa_{0}:=\frac{2 N+2}{g_{0}}$. Then, the $q_{0}$-breather sequence of modes $\left\{\bar{q}_{k}\right\}$ coincides with Fix $\left(R^{\kappa_{0}}\right) \cap F i x(S)$ considered as an invariant submanifold of Fix $(S)$.

proof: Firstly, following 32 , we recall that

$$
\begin{aligned}
\text { Fix }\left(R^{\kappa_{0}}\right) & =\left\{Q_{j}=P_{j}=0 \text { iff } j \neq 0 \bmod \left(g_{0}\right)\right\} \\
F i x(S) & =\left\{Q_{j}=-Q_{2 N+2-j}, P_{j}=-P_{2 N+2-j}\right\}
\end{aligned}
$$

so that it is possible to interpret $F i x(S)$ as the embedding of the fixed boundary condition system in the periodic boundary condition system. The thesis reduces to

$$
\left\{j=0 \bmod \left(g_{0}\right)\right\}=\left\{\bar{q}_{k}\right\}, \quad j=1, \ldots, N .
$$

In order to show the double inclusion of the two sets it is useful to remind that, from the definition of $g_{0}$, it follows

$$
\alpha q_{0}+\beta(2 N+2)= \pm 1 \quad \alpha, \beta \in \mathbb{Z} .
$$

$\subseteq$ ) let us take $j=0 \bmod \left(g_{0}\right)$, which means $j=J g_{0}$ with $J \leq\left[\frac{N+1}{g_{0}}\right]$; we have that

$$
A q_{0}+B(2 N+2)= \pm J g_{0}, \quad A=\alpha J, B=\beta J
$$

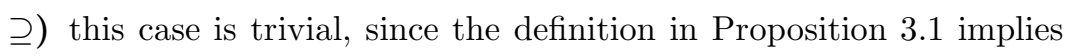
$g_{0} / \bar{q}_{k}$.

Proposition 5.1 claims that we should interpret the fixed boundary conditions system as embedded in the periodic one and then consider the symmetries Fix $\left(R^{\kappa}\right)$ with $2(N+1)=\kappa g$; observe that it must be $\kappa \neq 2$, otherwise $q_{0}=N+1>N$. So, any QB is a special orbit of one of such submanifolds. Observe also that, for $\kappa=2 N+2$ the invariant submanifold coincides with the fixed boundary subsystem Fix $\left(R^{\kappa}\right) \cap$ $F i x(S)=F i x(S)$, while for $\kappa=2$ we have $F i x\left(R^{\kappa}\right) \cap F i x(S)=\{0\}$. All the others submanifolds $F i x\left(R^{\kappa}\right) \cap F i x(S)$ with $\kappa$ not being a divisor of $2 N+2$ are meaningless since

Proposition 5.2 For all $l=1, \ldots, 2 N+2$ holds true

$$
F i x\left(R^{l}\right)=F i x\left(R^{\operatorname{gcd}(l, 2 N+2)}\right) .
$$

proof: Simply, by calling $\kappa=\operatorname{gcd}(l, 2 N+2)$ and $2 N+2=\kappa g$

$$
\frac{2 N+2}{\operatorname{gcd}(l, 2 N+2)}=g=\frac{2 N+2}{\operatorname{gcd}(\kappa, 2 N+2)} \text {. }
$$


The final scenario is the following. Take $1 \leq q_{0} \leq N$ and $g_{0}, \kappa_{0}$ ad defined in the Proposition 5.1, then

1. $g_{0}=1 \Leftrightarrow \kappa_{0}=2 N+2 \Leftrightarrow \operatorname{Fix}\left(R^{\kappa_{0}}\right) \cap \operatorname{Fix}(S)=\operatorname{Fix}(S)$ : the submanifold is trivial.

2. $g_{0} \geq 2$ and $\frac{2 N+2}{g_{0}} \notin \mathbb{N} \Rightarrow$ the submanifold doesn't coincide with Fix $(S)$ but cannot represent a fixed boundary conditions subsystem. Hence, the QB does not involve all the modes (not trivial), but it is not a rescaled solution of a lower dimensional system.

3. $g_{0} \geq 2$ and $g_{0}=\operatorname{gcd}\left(N+1, q_{0}\right) \Rightarrow$ the submanifold is the embedding of a fixed boundary condition subsystem with $\frac{N+1}{g_{0}}$ particles and the $\mathrm{QB}$ is the $\frac{q_{0}}{g_{0}}$-mode rescaled solution, constructed as in 25.

\subsection{Appendix C.}

It is known (cfr. 20]) that the sequence

$$
\left\{\begin{array}{l}
\lambda_{1}=1 \\
\lambda_{n}=\sum_{j=1}^{n-1} \lambda_{j} \lambda_{n-j}=\sum_{i+j=n} \lambda_{i} \lambda_{j}
\end{array}\right.
$$

is equivalent to a suitable functional equation

$$
g(z)=z+g^{2}(z), \quad g(z)=\sum_{r \geq 1} \lambda_{r} z^{r}
$$

and that follows $\lambda_{r}<4^{r-1}$. Consider now the modified problem

$$
\left\{\begin{array}{l}
\lambda_{1}=\mu_{1} \\
\lambda_{n}=\mu_{n} \sum_{j=1}^{n-1} \lambda_{j} \lambda_{n-j}
\end{array}\right.
$$

where $\mu_{j}$ is a limited sequence. Let $\mu:=\sup _{j} \mu_{j}$ and define the auxiliary sequence

$$
\left\{\begin{array}{l}
\Lambda_{1}=\mu \\
\Lambda_{n}=\mu \sum_{j=1}^{n-1} \Lambda_{j} \Lambda_{n-j}
\end{array},\right.
$$

whose related function satisfies

$$
g(z)=\mu z+\mu g^{2}(z)
$$

It is easy to prove by induction that

$$
\lambda_{n}=\mu_{n} \sum_{j=1}^{n-1} \lambda_{j} \lambda_{n-j} \leq \mu \sum_{j=1}^{n-1} \Lambda_{j} \Lambda_{n-j}=\Lambda_{n} .
$$

Thus, a bound for $\lambda_{n}$ can be obtained from the following asymptotic behaviour of $\Lambda_{n}$

$$
\Lambda_{n}=\mu\left(2 \mu^{2}\right)^{n-1} \frac{(2 n-3) ! !}{n !} \sim \frac{\mu}{n}\left(4 \mu^{2}\right)^{n-1} .
$$


This result can be applied to Theorem 3.1 where we deal with the sequence

$$
c_{n}:=\omega_{n q_{0}} \sum_{l=1}^{n-1} \omega_{l q_{0}} \omega_{(n-l) q_{0}} c_{l} c_{n-l}
$$

which reads also

$$
\lambda_{n}:=\omega_{n q_{0}}^{2} \sum_{l=1}^{n-1} \lambda_{l} \lambda_{n-l}
$$

where $\lambda_{l}=\omega_{l q_{0}} c_{l}$ and $\omega_{l}^{2}<4$.

Let us move to a more complex sequence

$$
\left\{\begin{array}{l}
\lambda_{1}=1 \\
\lambda_{n}=\sum_{i+j+k=n+1} \lambda_{i} \lambda_{j} \lambda_{k}
\end{array}\right.
$$

where plainly $1 \leq i, j, k \leq n-1$. Following [2, we define an analytic function $g(z)=\sum_{r \geq 1} \lambda_{r} z^{r}$ such that (37) is equivalent to the functional equation

$$
g=z+\frac{1}{z} g^{3}
$$

we stress that, since $g$ has a first order zero in $z=0$, then also $\frac{1}{z} g(z)$ is analytic. Let us define an auxiliary function $f(z)=\frac{1}{z} g(z)=$ $\sum_{r \geq 0} c_{r} z^{r}$, with $c_{r}=\lambda_{r+1}$, and rewrite (38) as

$$
f=1+z f^{3}, \quad f(0)=c_{0}=\lambda_{1}=1 .
$$

The differentiation of (39) gives

$$
f^{\prime}=\frac{f^{3}}{1-3 z f^{2}}
$$

which, exploiting again (39), reads as a $z$-independent expression

$$
f^{\prime}=\frac{f^{4}}{3-2 f} .
$$

We may assume the following general description for $f^{(r-1)}$

$$
f^{(r-1)}=\sum_{j=0}^{2 r-3} \sum_{k=0}^{4 r} \beta_{j k} \frac{f^{k}}{(3-2 f)^{j}}
$$

with suitable values for $\beta_{j k}$, and check its reliability by induction. The differentiation of the generic addendum in (41) gives

$$
\frac{d}{d z} \frac{f^{k}}{(3-2 f)^{j}}=\frac{k f^{k+3}}{(3-2 f)^{j+1}}+\frac{2 j f^{k+4}}{(3-2 f)^{j+2}},
$$

which yields

$$
f^{(r)}=\sum_{j=0}^{2 r-1} \sum_{k=0}^{4 r+4} \alpha_{j k} \frac{f^{k}}{(3-2 f)^{j}}
$$


where the new coefficients $\alpha_{j k}$ are properly defined through $\beta_{j k}$. As noticed in [2], Lemma 11.2, we can avoid the computations of $\alpha_{j k}$ simply using the previous computation

$$
\begin{aligned}
f^{(r)}(0) & =\sum_{j=0}^{2 r-3} \sum_{k=0}^{4 r} \beta_{j k} \frac{k f(0)^{k+3}}{(3-2 f(0))^{j+1}}+\frac{2 j f(0)^{k+4}}{(3-2 f(0))^{j+2}}= \\
& =\sum_{j=0}^{2 r-3} \sum_{k=0}^{4 r} \beta_{j k}(k+2 j)
\end{aligned}
$$

which allows to find

$$
c_{r}=\frac{1}{r !} f^{(r)}(0)<\frac{1}{r !} 8 r \sum_{j=0}^{2 r-3} \sum_{k=0}^{4 r} \beta_{j k}=8 r \frac{1}{r !} f^{(r-1)}(0)=8 c_{r-1} .
$$

This concludes the proof, since we have finally shown

$$
\lambda_{n} \leq 8^{n-1} .
$$

Let us consider the sequence

$$
\left\{\begin{array}{l}
\mu_{1}=1, \\
\mu_{2}=1 \\
\mu_{n}=\sum_{i+j+k=n} \mu_{i} \mu_{j} \mu_{k}
\end{array} .\right.
$$

Here the solution comes from the comparison of (43) with the previous sequence (37). Indeed, with the choice $\lambda_{1}=\mu_{1}=\mu_{2}=1$, it is possible to prove by induction that $\mu_{n} \leq \lambda_{n-1} \leq \lambda_{n}$. 


\section{References}

[1] Bambusi D. and Ponno A., On meta-stability in FPU, Comm. Math. Phys, 264 (2005), pp. 539-561.

[2] Benettin G., Galgani L. and Giorgilli A., Realization of holonomic constraints and freezing of high frequency degrees of freedom in the light of classical perturbation theory. II, Comm. Math. Phys, 121, 557-601 (1989).

[3] Berchialla L., Galgani L. And Giorgilli A., Localization of energy in FPU chains, Discrete Contin. Dyn. Syst., 11 (2004), pp. 855-866.

[4] Berchialla L., Giorgilli A. and Paleari S., Exponentially long times to equipartition in the thermodynamic limit, Phys. Lett. A, 321 (2004), pp. 167-172.

[5] Bocchieri P., Scotti A., Bearzi B., and Loinger A., Anharmonic chain with Lennard-Jones interaction, Phys. Rev. A, 2 (1970), pp. 2013-2019.

[6] Carati A., Galgani L., Ponno A., and Giorgilli A., The Fermi-Pasta-Ulam problem, Nuovo Cimento B (11), 117 (2002), pp. 1017-1026.

[7] Casetti L., Cerruti-Sola M., Pettini M., and E. G. D. Cohen, The Fermi-Pasta-Ulam problem revisited: stochasticity thresholds in nonlinear Hamiltonian systems, Phys. Rev. E (3), 55 (1997), pp. 6566-6574.

[8] Cercignani C., Galgani L., and Scotti A., Phys. Lett. A, 38 (1972), p. 403.

[9] Conway J.H. and Jones A.J., Trigonometric diophantine equations (On vanishing sums of roots of unity), Acta Arithmetica, XXX (1976), pp. 229-240.

[10] De Luca J., Lichtenberg A., and Lieberman M.A., Time scale to ergodicity in the fermi-pasta-ulam system, Chaos, 5 (1995), pp. 283-297.

[11] De Luca J., Lichtenberg A., and Ruffo S., Energy transition and time scale to equipartition in the fermi-pasta-ulam oscillator chain, Phys. Rev. E (3), 51 (1995), pp. 2877-2884

[12] - Finite times to equipartition in the thermodynamic limit, Phys. Rev. E (3), 60 (1999), pp. 3781-3786.

[13] Dvornicich R. And Zannier U., On sums of roots of unity, Monatsh. Math., 129.2 (2000), pp. 97-108.

[14] Fermi E., Pasta J. and Ulam S., Los Alamos report L.A.1940, Enrico Fermi, Collected Papers II (1955), Univ. Chicago Press, Chicago (1965), pp. 977-988.

[15] Flach S. and Gorbach A.,Discrete breathers in Fermi-PastaUlam lattices, Chaos, 15 (2005), pp. 015112 (10). 
[16] Flach S., Ivanchenko V. and Kanakov O.I., q-breathers and the Fermi-Pasta-Ulam problem, Phys. Rev. Lett. 95 (2005), 064102; q-Breathers in Fermi-Pasta-Ulam chains: existence, localization and stability, Phys. Rev. E 73 (2996), 036618.

[17] Fucito F., Marchesoni F., Marinari E., Parisi G., Peliti L., Ruffo S., And Vulpiani A., Approach to equilibrium in a chain of nonlinear oscillators, J. Physique, 43 (1982), pp. 707713.

[18] Galgani L. And Scotti A., Phys. Rev. Lett., 28 (1972), p. 1173.

[19] —, Recent progress in classical nonlinear dynamics, Riv. Nuovo Cimento (2), 2 (1972), pp. 189-209.

[20] Giorgilli A. And Muraro D., Exponentially stable manifolds in the neighbourhood of elliptic equilibria.

[21] Giorgilli A., Paleari S. and Penati T., Local chaotic behaviour in the FPU system, Discrete Contin. Dyn. Syst. Ser. B, 5 (2005), pp. 991-1004.

[22] Ivanchenko V., Kanakov O.I., Mishagin K.G. and Flach S., q-Breathers in Finite Two- and Three-Dimensional Nonlinear Acoustic Lattices, Phys. Rev. Lett. 97 (2006) 025505.

[23] Izrailev F. M. And Chirikov B. V., Statistical properties of a nonlinear string (Institute of Nuclear Physics, Novosibirsk, USSR, 1965) (in Russian).

[24] Izrailev F. M. and Chirikov B. V., Stochasticity of the simplest dynamical model with divided phase space, Sov. Phys. Dokl., 11 (1966), p. 30.

[25] Kanakov O.I., Flach S., Ivanchenko V. and Mishagin K. G., Scaling properties of q-breather in nonlinear acoustic lattices, nlin.PS/0607019 (2006).

[26] Lorenzoni P. And Paleari S., Metastability and dispersive shock waves in Fermi-Pasta-Ulam system., Physica D, to appear.

[27] Paleari S. and Penati T., Relaxation time to equilibrium in Fermi-Pasta-Ulam system, in Symmetry and perturbation theory (Cala Gonone, 2004), World Sci. Publishing, River Edge, NJ, 2004, pp. 255-263.

[28] — Equipartition times in a Fermi-Pasta-Ulam system, Discrete Contin. Dyn. Syst., (2005). Dynamical systems and differential equations (Pomona, CA, 2004).

[29] Poggi P. And Ruffo S. Exact solutions in the FPU oscillator chain, Phys. D, 103 (1997), pp. 251-272.

[30] Ponno A. Scaling laws of the energy cascade, Cargese Proceeding.

[31] RINk B. Symmetry and resonance in periodic FPU chains., Comm. Math. Phys., 218 (2001), pp. 665-685.

[32] RINk B.Symmetric invariant manifolds in the Fermi-Pasta-Ulam lattice., Physica D, 175 (2003), pp. 31-42. 
[33] RINK B.Proof of Nishida's conjecture on anharmonic lattices., (2005) preprint

[34] Shepelyansky D.L., Low-energy chaos in the Fermi-Pasta-Ulam problem, Nonlinearity, 10 (1997), pp. 1331-1338.

[35] Ullmann K., Lichtenberg A., And Corso G., Energy equipartition starting from high-frequency modes in the FermiPasta-Ulam $\beta$ oscillator chain, Phys. Rev. E (3), 61 (2000), pp. 2471-2477. 


\section{List of captions}

1. FIG.1: (a): Natural packet evolution initially exciting mode $q_{0}=1$. The energies of normal modes are plotted versus $q$. Circles - $10^{4}$, squares - $10^{5}$, rhombs - $10^{6}$. Dashed line - $q$-breather from (b) for comparison. (b): The energies of normal modes versus $q$ for a $q$-breather with $q_{0}=1$. (c): sequence $\eta_{j}^{-1}$. (d): sequence $\chi_{j}^{-1}$. Parameters in all cases: $N=31, \alpha=0.33, \mathcal{E}=$ 0.01 .

2. FIG.2: (a): Natural packet evolution initially exciting mode $q_{0}=2$ (circles). The energies of normal modes are plotted versus $q$ at $t=10^{5}$. The dashed line shows the energy distribution for a corresponding $q$-breather with $q_{0}=2$. (b): Same as (a), but for $q_{0}=3$. (c): sequence $\eta_{j}^{-1}(2,47)$. (d): sequence $\eta_{j}^{-1}(3,47)$. Parameters in all cases: $N=47, \alpha=0.1, \mathcal{E}=0.0025$.

3. FIG.3: Parameters: $N=47, \alpha=0.1, \mathcal{E}=0.0025$. (a): Energy of normal modes versus mode number of a QB for $q_{0}=2$. (b): Similar to (a), but for a QB with $q_{0}=3$. (c): sequence $\chi_{j}^{-1}(2,47)$. (d): sequence $\chi_{j}^{-1}(3,47)$.

4. FIG.4: Parameters: $N=47, \mathcal{E}=0.1$. (a): Energy of normal modes in a QB (open symbols) and a natural packet (filled symbols, waiting time $10^{5}$ ) for $q_{0}=47$ and $\alpha=0.25$. (b): Similar to (a), but for $\beta=0.25$. (c): sequence $\chi_{j}^{-1}$ which corresponds to case (a). (d): sequence $\chi_{j}^{-1}$ which corresponds to (b). 